\title{
Noncommutative generalized Gibbs ensemble in isolated integrable quantum systems
}

\author{
Kouhei Fukai $\oplus^{*}$, Yuji Nozawa $\odot$, Koji Kawahara $\odot$, and Tatsuhiko N. Ikeda ${ }^{\dagger}$ \\ The Institute for Solid State Physics, The University of Tokyo, Kashiwa, Chiba 277-8581, Japan
}

(Received 5 March 2020; accepted 19 August 2020; published 14 September 2020)

\begin{abstract}
The generalized Gibbs ensemble (GGE), which involves multiple conserved quantities other than the Hamiltonian, has served as the statistical-mechanical description of the long-time behavior for several isolated integrable quantum systems. We argue that the GGE may involve a noncommutative set of conserved quantities in view of the maximum entropy principle and show that the GGE thus generalized (noncommutative GGE, NCGGE) gives a more qualitatively accurate description of the long-time behaviors than that of the conventional GGE. Providing a clear understanding of why the (NC)GGE well describes the long-time behaviors, we construct, for noninteracting models, the exact NCGGE that describes the long-time behaviors without an error even at finite system size. It is noteworthy that the NCGGE involves nonlocal conserved quantities, which can be necessary for describing long-time behaviors of local observables. We also give some extensions of the NCGGE and demonstrate how accurately they describe the long-time behaviors of few-body observables.
\end{abstract}

DOI: 10.1103/PhysRevResearch.2.033403

\section{INTRODUCTION}

The foundation of quantum statistical mechanics has seen a resurgence of interest in recent years [1-4] partly because well-isolated and -controlled artificial quantum systems have emerged as the ideal platform to reconsider the long-standing problem [5-10]. A remarkable finding is that an isolated quantum many-body system can relax to an effective stationary state even without energy dissipation or quantum decoherence. Although the stationary state in the strict sense appears only in infinite systems, an effective (or approximate) stationary state arises at large but finite system sizes, where the fluctuations and recurrences are negligible [11-13].

In generic nonintegrable systems, the effective stationary state coincides in fact with the thermal state due to the eigenstate thermalization hypothesis (ETH) [14,15], which dates back to von Neumann [16] and has recently been numerically verified [17-23]. Meanwhile, there exist known systems in which the stationary state does not coincide with the thermal state such as integrable systems [18,24-27], many-body localized systems [28,29], and so on [30-32]. It remains an open question how to classify all the nonthermal systems and to identify the statistical-mechanical ensemble describing those states.

The generalized Gibbs ensemble (GGE) is a paradigmatic framework to describe various nonthermal stationary states [24]. Whereas the usual Gibbs (canonical) ensemble involves

\footnotetext{
*k.fukai@issp.u-tokyo.ac.jp

†tikeda@issp.u-tokyo.ac.jp

Published by the American Physical Society under the terms of the Creative Commons Attribution 4.0 International license. Further distribution of this work must maintain attribution to the author(s) and the published article's title, journal citation, and DOI.
}

the Hamiltonian, the GGE does other conserved quantities as well [see Eq. (5) below] [33]. The GGE describes the stationary states in noninteracting integrable models (hard-core bosons [24], the transverse-field Ising model [34]), interacting (Bethe-ansatz) integrable ones [35-40], models with different-type conserved quantities [41], quantum field theories [42], and so on $[43,44]$.

Despite its success, the GGE sometimes fails to describe the stationary state. For example, spinless fermions or hard-core bosons under quasiperiodic potential cannot be described by the GGE due to the localization of single-particle eigenstates [45-49]. Another example is the entanglement prethermalization in an interacting integrable system [50], where nonlocal conserved quantities play significant roles. One crucial problem is that the GGE is a general framework and never tells us which conserved quantities should be incorporated. When a GGE fails, it is hard to tell whether the ad hoc set of conserved quantities is not enough or the framework breaks down. In particular, the GGEs mentioned above implicitly assume that the conserved quantities commute with each other (commutative GGE, CGGE), and this assumption may unnecessarily constrain the GGE. The GGE conserved quantities can be noncommutative in view of the maximum entropy principle. The GGE with a noncommutative set of conserved quantities was first introduced in Ref. [51] in discussing the prerelaxation for the XY spin chain. The ensemble with a noncommutative set of conserved quantities was also mentioned in Ref. [52]. However, it has not been systematically studied why and how those GGEs describe local or few-body observables well.

In this paper, we systematically study how the additional noncommutative conserved quantities affect the GGE and show that the GGE thus generalized (noncommutative GGE, NCGGE) describes the stationary states in isolated integrable systems better than the conventional CGGE. By introducing the observable projection idea, we provide a clear 
understanding of why the (NC)GGE well describes the stationary states. In this spirit, for a noninteracting model, we systematically construct the NCGGE that describes the stationary states without an error at finite system size for few-body observables. We also propose some extensions of the NCGGE and demonstrate how they work.

The rest of this paper is organized as follows. In Sec. II, we formulate the problem and define the NCGGE. In Sec. III, we explain why the GGE is valid with enough conserved quantities and the necessity of the NCGGE. The observable projection idea and the uniqueness of the NCGGE presented in Secs. II and III are so general that they can be applied to both interacting and noninteracting integrable systems. In Secs. IV and V, focusing on free fermions, we show more detailed analyses of the NCGGE. In Sec. IV, we give the example of NCGGE in free fermion and show the exactness of NCGGE even at finite system size. In Sec. V, we give numerical results for two-body observables in the CGGE and NCGGE. In Sec. VI, we give further extensions of the NCGGE. Finally, in Sec. VII, we summarize our study with concluding remarks.

\section{FORMULATION OF PROBLEM AND NCGGE}

We consider an isolated quantum system described by a time-independent Hamiltonian $\hat{H}$. We let $\left\{E_{n}\right\}$ denote the distinct eigenenergies, having $\hat{H}=\sum_{m} E_{m} \hat{P}_{m}$ with $\hat{P}_{m}$ being the projection operator onto the corresponding eigenspace. Under the Hamiltonian, an initial state $\left|\psi_{\text {ini }}\right\rangle$ evolves as $|\psi(t)\rangle=$ $e^{-i H t}\left|\psi_{\text {ini }}\right\rangle=\sum_{m} e^{-i E_{m} t} \hat{P}_{m}\left|\psi_{\text {ini }}\right\rangle$ at time $t \quad(\hbar=1$ throughout this paper). Assuming that $\left|\psi_{\text {ini }}\right\rangle$ is a superposition of exponentially-large number (in terms of the system size) of energy eigenstates [12,53,54], we have an effective stationary state, in which an observable $\hat{A}$ has its expectation value equal to the long-time average

$$
\langle\hat{A}\rangle_{\mathrm{LT}}=\overline{\langle\psi(t)|\hat{A}| \psi(t)\rangle}=\sum_{m}\left\langle\psi_{\mathrm{ini}}\left|\hat{P}_{m} \hat{A} \hat{P}_{m}\right| \psi_{\mathrm{ini}}\right\rangle,
$$

where $\overline{f(t)} \equiv \lim _{T \rightarrow \infty} \int_{0}^{T}(d t / T) f(t)$. It is convenient to define the diagonal and off-diagonal decomposition of $\hat{A}$ by $\hat{A}=$ $\hat{\bar{A}}+\delta \hat{A}$ with $\hat{\bar{A}} \equiv \sum_{m} \hat{P}_{m} \hat{A} \hat{P}_{m}$ and $\delta \hat{A} \equiv \sum_{m, n(m \neq n)} \hat{P}_{m} \hat{A} \hat{P}_{n}$. This notation simplifies Eq. (1) as

$$
\langle\hat{A}\rangle_{\mathrm{LT}}=\langle\hat{\bar{A}}\rangle_{\mathrm{ini}} \equiv\left\langle\psi_{\mathrm{ini}}|\hat{\bar{A}}| \psi_{\mathrm{ini}}\right\rangle
$$

If $\hat{A}$ is a conserved quantity $\hat{Q}$, i.e. $[\hat{Q}, \hat{H}]=0$, Eq. (1) leads to

$$
\langle\hat{Q}\rangle_{\mathrm{LT}}=\langle\hat{Q}\rangle_{\mathrm{ini}}
$$

Equation (1) gives $\langle\hat{A}\rangle_{\mathrm{LT}}$ exactly but involves an exponentially-large number of inputs corresponding to every detail of $\left|\psi_{\text {ini }}\right\rangle$. The question that we address in this paper is to find a statistical-mechanical ensemble $\hat{\rho}$ which, with fewer (up to a polynomially-large number of) inputs, satisfies $\langle\hat{A}\rangle_{\mathrm{LT}} \simeq \operatorname{Tr}(\hat{\rho} \hat{A})$ for local or few-body observables $\hat{A}$ 's of interest. Here, $\simeq$ allows an error due to the finite-size effect that vanishes in the thermodynamic limit.

The GGE is a successful candidate for such an ensemble formulated as follows. The central idea is that the ensemble $\hat{\rho}$ would maximize the von Neumann entropy $S(\hat{\rho})=$
$-\operatorname{Tr}(\hat{\rho} \ln \hat{\rho})$ (the Boltzmann constant is set to unity). When there exist multiple conserved quantities $\left\{\hat{Q}_{\alpha}\right\}$ including the Hamiltonian, the dynamics is constrained by Eq. (3) for each $\hat{Q}=\hat{Q}_{\alpha}$. Then the ensemble that maximizes the entropy under the constraints is given by the stationary condition for

$$
\Psi\left(\hat{\rho},\left\{\lambda_{\alpha}\right\}\right)=S(\hat{\rho})-\sum_{\alpha} \lambda_{\alpha}\left[\operatorname{Tr}\left(\hat{\rho} \hat{Q}_{\alpha}\right)-\left\langle\hat{Q}_{\alpha}\right\rangle_{\text {ini }}\right],
$$

with the Lagrange multipliers $\left\{\lambda_{\alpha}\right\}$. This condition leads to [33]

$$
\hat{\rho}_{\mathrm{GGE}}=\frac{e^{-\sum_{\alpha} \lambda_{\alpha} \hat{Q}_{\alpha}}}{Z},
$$

where $Z \equiv \operatorname{Tr} e^{-\sum_{\alpha} \lambda_{\alpha} \hat{Q}_{\alpha}}$ is the partition function and the Lagrange multipliers $\left\{\lambda_{m}\right\}$ called the generalized temperatures are determined uniquely by

$$
\left\langle\hat{Q}_{\alpha}\right\rangle_{\mathrm{ini}}=\operatorname{Tr}\left(\hat{\rho}_{\mathrm{GGE}} \hat{Q}_{\alpha}\right),
$$

for each $\alpha$. When $\left\{\hat{Q}_{\alpha}\right\}$ consists only of the Hamiltonian, the GGE reduces to the usual Gibbs (canonical) ensemble and the generalized temperature is the inverse temperature $\beta$. Once determined, the GGE gives expectation values for generic observables by $\langle\hat{A}\rangle_{\mathrm{GGE}} \equiv \operatorname{Tr}\left(\hat{\rho}_{\mathrm{GGE}} \hat{A}\right)$.

We emphasize that, in deriving Eq. (5), we never use the commutativity $\left[\hat{Q}_{\alpha}, \hat{Q}_{\beta}\right]=0$, which is implicitly assumed in the literature. In the Heisenberg model, for example, the SU(2) symmetry implies that each of the total $S^{x}, S^{y}$, and $S^{z}$ is a conserved quantity, and one can construct the GGE by using all of them. Thus allowing noncommutative ones increases the number of conserved quantities and improves the GGE in general.

We note that, when $\left[\hat{Q}_{\alpha}, \hat{Q}_{\beta}\right] \neq 0$, we cannot decompose Eq. (5) into the exponentials for each conserved quantity: $e^{-\sum_{\alpha} \lambda_{\alpha} \hat{Q}_{\alpha}} \neq \prod_{\alpha} e^{-\lambda_{\alpha} \hat{Q}_{\alpha}}$. Nevertheless, the exponential $e^{-\sum_{\alpha} \lambda_{\alpha} \hat{Q}_{\alpha}}$ is well-defined and the generalized temperatures are uniquely determined. We prove these facts in the Appendix A.

\section{VALIDITY OF NCGGE IN THERMODYNAMIC LIMIT}

Before discussing concrete models, we show why the GGE well describes the long-time behaviors (1) for generic observables in the thermodynamic limit. Although the GGE is usually justified by the generalized ETH [55], we here provide another perspective, in which the merit of the NCGGE becomes evident.

To justify the GGE, we invoke the observable projection with conserved quantities [56]. Note that the Hilbert-Schmidt inner product can be defined between two traceless observables $\hat{A}$ and $\hat{B}$ as $\langle\hat{A}, \hat{B}\rangle \equiv \operatorname{Tr}(\hat{A} \hat{B}) / D$ with $D$ being the Hilbert-space dimension. For a given orthogonal set of conserved quantities $\left\{\hat{Q}_{\alpha}\right\}$, we can decompose the observable $\hat{A}$ into the parallel and perpendicular components: $\hat{A}=\hat{A}_{\|}+$ $\hat{A}_{\perp}$, where $\hat{A}_{\|}=\sum_{\alpha} p_{A \alpha} \hat{Q}_{\alpha}$ and $p_{A \alpha} \equiv\left\langle\hat{A}, \hat{Q}_{\alpha}\right\rangle /\left\langle\hat{Q}_{\alpha}, \hat{Q}_{\alpha}\right\rangle$. We call our $\left\{\hat{Q}_{\alpha}\right\}$ a "complete" set of conserved quantities when the diagonal component $\hat{\bar{A}}_{\perp}$, which is relevant in the long-time average, vanish in the thermodynamic limit.

The observable projection idea readily justifies the GGE in the thermodynamic limit as follows. Note that the 
long-time average for the actual dynamics is $\langle\hat{A}\rangle_{\mathrm{LT}}=$ $\left\langle\hat{A}_{\|}\right\rangle_{\mathrm{LT}}+\left\langle\hat{A}_{\perp}\right\rangle_{\mathrm{LT}}=\left\langle\hat{A}_{\|}\right\rangle_{\text {ini }}+\left\langle\hat{\bar{A}}_{\perp}\right\rangle_{\text {ini }}$, where we have used $\hat{A}_{\|}=\sum_{\alpha} p_{A \alpha} \hat{Q}_{\alpha}$ and Eqs. (2) and (3). On the other hand, the GGE gives $\langle\hat{A}\rangle_{\mathrm{GGE}}=\left\langle\hat{A}_{\|}\right\rangle_{\mathrm{GGE}}+\left\langle\hat{A}_{\perp}\right\rangle_{\mathrm{GGE}}=\left\langle\hat{A}_{\|}\right\rangle_{\mathrm{ini}}+$ $\left\langle\hat{\bar{A}}_{\perp}\right\rangle_{\mathrm{GGE}}$ since the GGE satisfies $\left\langle\hat{Q}_{\alpha}\right\rangle_{\mathrm{ini}}=\left\langle\hat{Q}_{\alpha}\right\rangle_{\mathrm{GGE}}$ by definition and then $\left\langle\hat{A}_{\|}\right\rangle_{\text {ini }}=\left\langle\hat{A}_{\|}\right\rangle_{\mathrm{GGE}}$ and $\left\langle\delta \hat{A}_{\perp}\right\rangle_{\mathrm{GGE}}=0$. Thus the error of the GGE description depends only on the perpendicular component as

$$
\langle\hat{A}\rangle_{\mathrm{LT}}-\langle\hat{A}\rangle_{\mathrm{GGE}}=\left\langle\hat{\bar{A}}_{\perp}\right\rangle_{\mathrm{ini}}-\left\langle\hat{\bar{A}}_{\perp}\right\rangle_{\mathrm{GGE}},
$$

which vanishes in the thermodynamic limit if our $\left\{\hat{Q}_{\alpha}\right\}$ is complete. When the set of conserved quantities $\left\{\hat{Q}_{\alpha}\right\}$ is incomplete, $\hat{\bar{A}}_{\perp}$ does not vanish and the GGE prediction deviates from the long-time average in the thermodynamic limit unless $\hat{\bar{A}}_{\perp}$ is accidentally fit by the GGE, i.e., $\left\langle\hat{\bar{A}}_{\perp}\right\rangle_{\text {ini }}=\left\langle\hat{\bar{A}}_{\perp}\right\rangle_{\text {GGE }}$. In Appendix B, we show that the nonvanishing norm of $\hat{A}_{\perp}$ implies the existence of the additional local conserved quantities which should be incorporated into $\left\{\hat{Q}_{\alpha}\right\}$.

The above justification of the GGE highlights the importance of taking enough amount of conserved quantities. In performing the operator projections, we can also single out relevant conserved quantities for the local observables of interest. We note that the role of noncommutative conserved quantities has not been clear in the above discussion, and it often happens that we only have commutative conserved quantities. In Appendix C, we show how to judge whether noncommutative ones need to be incorporated into the GGE or not.

Finite-size systems are also of interest, in which the long-time average can be influenced by nonlocal conserved quantities, which are excluded from the minimal complete set of the conserved quantities in the thermodynamic limit. Incorporating those conserved quantities, we have smaller errors with GGEs at finite system size or more accurate GGEs.

We can apply all the arguments in this section to any system including interacting integrable systems and even nonintegrable systems. Upon calculating $\hat{\bar{A}}$ and its parallel and perpendicular components, we need to perform numerically the exact diagonalization of $\hat{H}$ and inner products between observables and conserved quantities with their explicit matrix representations. Thus the accessible system size is rather limited (see also Sec. VII for further discussion). On the other hand, in noninteracting integrable systems, we can do more analytically to get deeper insights, and larger system sizes are accessible. In the following, we focus on free fermions in one dimension and discuss various versions of the NCGGE.

\section{EXACT NCGGE AT FINITE SYSTEM SIZE}

Interestingly, for free fermions in one dimension, we can analytically construct NCGGE which exactly describes the long-time average at finite system size. The construction is step-by-step: The NCGGE involving all the up-to- $N$-body conserved quantities exactly describes all the up-to- $N$-body observables.
We begin by defining the model Hamiltonian

$$
\hat{H}=-\sum_{i=1}^{L}\left(\hat{c}_{i}^{\dagger} \hat{c}_{i+1}+\text { H.c. }\right)=\sum_{k} \epsilon_{k} \hat{c}_{k}^{\dagger} \hat{c}_{k},
$$

where we have set the transfer integral to unity, $L$ is the number of sites, the periodic boundary condition is imposed, and $\hat{c}_{i}\left(\hat{c}_{i}^{\dagger}\right)$ is the annihilation (creation) operator for the spinless fermion at site $i:\left\{\hat{c}_{i}, \hat{c}_{j}^{\dagger}\right\}=\delta_{i j}$ and $\left\{\hat{c}_{i}, \hat{c}_{j}\right\}=\left\{\hat{c}_{i}^{\dagger}, \hat{c}_{j}^{\dagger}\right\}=0$ for all $i$ and $j$. We have introduce the Fourier transform $\hat{c}_{k}=$ $L^{-1 / 2} \sum_{j} e^{-i k j} \hat{c}_{j}$ and $\epsilon_{k}=-2 \cos k$, where $k=2 \pi m / L(m \in$ $\mathbb{Z})$ with $-\pi<k \leqslant \pi$. Thus $\sum_{k}$ means the sum over the range $\{2 \pi m / L \mid-L / 2<m \leqslant L / 2, m \in \mathbb{Z}\}$.

At one-body level, this Hamiltonian has two kinds of conserved quantities:

$$
\hat{I}_{k}=\hat{c}_{k}^{\dagger} \hat{c}_{k}, \quad \hat{J}_{k}=\hat{c}_{-k}^{\dagger} \hat{c}_{k} .
$$

While only $\hat{I}_{k}$ is usually considered in the literature [24], $\hat{J}_{k}$ arising from the double degeneracy of the dispersion relation in the single-particle spectrum $\epsilon_{k}=\epsilon_{-k}$ except $k=0$ and $\pi$ (similarly to the XY chain case in Ref. [51]) is also allowed in the NCGGE. The set of these conserved quantities are nonconmmutative due to the algebra $\left[\hat{I}_{k}, \hat{J}_{ \pm k}\right]=\mp \hat{J}_{ \pm k}$ and $\left[\hat{J}_{k}, \hat{J}_{-k}\right]=\hat{I}_{-k}-\hat{I}_{k}$ (all other commutators vanish).

Note that $\hat{I}_{k}$ can be written as the sum of local conserved quantities (see the supplemental material of Ref. [56]), but $\hat{J}_{k}$ cannot. Taking the Fourier transformation of $\hat{J}_{k}$, we have the Wannier-basis form of the additional conserved quantity $\hat{W}_{n}$,

$$
\hat{W}_{n} \equiv \sum_{k} e^{-i n k} \hat{J}_{k}=\sum_{j=1}^{L} \hat{c}_{j+n}^{\dagger} \hat{c}_{-j},
$$

where the site indices $j+n$ and $-j$ should be interpreted in modulo $L$. Note that $\hat{W}_{n}$ includes the long-range hopping of $O(L)$ and local hopping with the same weight, which implies that $\hat{J}_{k}$ is a nonlocal conserved quantity.

We define the GGE with all the one-body conserved quantities in Eq. (9) as the one-body NCGGE:

$$
\hat{\rho}_{1 \mathrm{NC}}=\frac{1}{Z_{1 \mathrm{NC}}} \exp \left[-\sum_{k}\left(\lambda_{k} \hat{I}_{k}+\omega_{k} \hat{J}_{k}\right)\right],
$$

where $Z_{1 \mathrm{NC}}=\operatorname{Tr} e^{-\sum_{k}\left(\lambda_{k} \hat{\hat{l}}_{k}+\omega_{k} \hat{\jmath}_{k}\right)}$ and $\lambda_{k}\left(=\lambda_{k}^{*}\right)$ and $\omega_{k}\left(=\omega_{-k}^{*}\right)$ are the generalized temperatures determined by

$$
\left\langle\hat{I}_{k}\right\rangle_{1 \mathrm{NC}}=\left\langle\hat{I}_{k}\right\rangle_{\mathrm{ini}}, \quad\left\langle\hat{J}_{k}\right\rangle_{1 \mathrm{NC}}=\left\langle\hat{J}_{k}\right\rangle_{\mathrm{ini}}
$$

for every $k$.

Remarkably, the one-body NCGGE thus constructed describe, without an error, long-time averages of all the one-body observables. To show this, we take an arbitrary one-body observable $\hat{A}^{(1)}=\sum_{k, q} A_{k q} \hat{c}_{k}^{\dagger} \hat{c}_{q}$ and consider its long-time average. Utilizing the Heisenberg picture, $\hat{c}_{k}(t) \equiv e^{i \hat{H} t} \hat{c}_{k} e^{-i \hat{H} t}=e^{-i \epsilon_{k} t} \hat{c}_{k}$ and $\hat{c}_{k}^{\dagger}(t)=e^{i \epsilon_{k} t} \hat{c}_{k}^{\dagger}$, we ob$\operatorname{tain}\left\langle\hat{A}^{(1)}\right\rangle_{\mathrm{LT}}=\sum_{k, q} A_{k q}\left\langle\hat{c}_{k}^{\dagger} \hat{c}_{q}\right\rangle_{\mathrm{ini}} \overline{e^{i\left(\epsilon_{k}-\epsilon_{q}\right) t}}=\sum_{k}\left(A_{k k}\left\langle\hat{I}_{k}\right\rangle_{\mathrm{ini}}+\right.$ $\left.A_{k,-k}\left\langle\hat{J}_{k}\right\rangle_{\text {ini }}\right)$. We emphasize that the long-time average has been nonvanishing only for $\epsilon_{k}=\epsilon_{q}$ and this condition is equivalent to that $\hat{c}_{k}^{\dagger} \hat{c}_{q}$ is a conserved quantity since $\left[\hat{H}, \hat{c}_{k}^{\dagger} \hat{c}_{q}\right]=\left(\epsilon_{k}-\epsilon_{q}\right) \hat{c}_{k}^{\dagger} \hat{c}_{q}$. On the other hand, we have, for the 
one-body NCGGE, $\left\langle\hat{A}^{(1)}\right\rangle_{1 \mathrm{NC}}=\sum_{k}\left(A_{k k}\left\langle\hat{I}_{k}\right\rangle_{\mathrm{ini}}+A_{k,-k}\left\langle\hat{J}_{k}\right\rangle_{\mathrm{ini}}\right)$ since $\left\langle\hat{c}_{k}^{\dagger} \hat{c}_{q}\right\rangle_{1 \mathrm{NC}}=0$ for $\epsilon_{k} \neq \epsilon_{q}$. Using Eq. (12), we obtain

$$
\left\langle\hat{A}^{(1)}\right\rangle_{1 \mathrm{NC}}=\left\langle\hat{A}^{(1)}\right\rangle_{\mathrm{LT}} \quad \forall \hat{A}^{(1)}
$$

even when the system size $L$ is finite. This is a remarkable property that the conventional CGGE does not have. The CGGE density matrix $\hat{\rho}_{\mathrm{C}}$ is defined only with $\hat{I}_{k}$ and cannot be exact at finite $L,\left\langle\hat{A}^{(1)}\right\rangle_{\mathrm{C}}=\sum_{k} A_{k k}\left\langle\hat{I}_{k}\right\rangle_{\mathrm{ini}} \neq\left\langle\hat{A}^{(1)}\right\rangle_{\mathrm{LT}}$.

The above exactness of the one-body NCGGE naturally let us find the exact $N$-body NCGGE. Let us first consider the $N=2$ case and take a two-body observable $\hat{A}^{(2)}=$ $\sum_{k_{1}, k_{2}, q_{1}, q_{2}} A_{k_{1} k_{2} ; q_{1} q_{2}} \hat{c}_{k_{1}}^{\dagger} \hat{c}_{k_{2}}^{\dagger} \hat{c}_{q_{2}} \hat{c}_{q_{1}}$. Its long-time average is given by $\left\langle\hat{A}^{(2)}\right\rangle_{\mathrm{LT}}=\sum_{k_{1}, k_{2}, q_{1}, q_{2}}^{\prime} A_{k_{1} k_{2} ; q_{1} q_{2}}\left\langle\hat{c}_{k_{1}}^{\dagger} \hat{c}_{k_{2}}^{\dagger} \hat{c}_{q_{2}} \hat{c}_{q_{1}}\right\rangle_{\text {ini }}$, where $\sum^{\prime}$ means the restriction of the sum to $\epsilon_{k_{1}}+\epsilon_{k_{2}}=\epsilon_{q_{1}}+\epsilon_{q_{2}}$. Here we note that every $\hat{C}_{k_{1} k_{2} q_{1} q_{2}} \equiv \hat{c}_{k_{1}}^{\dagger} \hat{c}_{k_{2}}^{\dagger} \hat{c}_{q_{2}} \hat{c}_{q_{1}}$ in the restricted sum is a conserved quantity. These two-body conserved quantities include the products of two one-body conserved quantities (9) as well as others due to accidental degeneracy such as $c_{\frac{\pi}{2}-k}^{\dagger} c_{\frac{\pi}{2}-q}^{\dagger} c_{\frac{\pi}{2}+q} c_{\frac{\pi}{2}+k}$. If we define the exact two-body NCGGE $\hat{\rho}_{2 \mathrm{NC}}$ by $\left\langle\hat{C}_{k_{1} k_{2} q_{1} q_{2}}\right\rangle_{2 \mathrm{NC}}=\left\langle\hat{C}_{k_{1} k_{2} q_{1} q_{2}}\right\rangle_{\text {ini }}$ and Eq. (12) for $2 \mathrm{NC}$, one can easily show $\left\langle\hat{A}^{(n)}\right\rangle_{2 \mathrm{NC}}=\left\langle\hat{A}^{(n)}\right\rangle_{\mathrm{LT}}\left(\forall \hat{A}^{(n)}\right)$ for $(n=1$ and 2). Thus we have obtained the NCGGE that describes the long-time average of each one- or two-body observable exactly at finite $L$. In a similar manner, we can systematically construct the exact $N$-body GGE that is exact for all up-to- $N$ body observables at finite system size. Note that the conserved quantities used in the $N$-body NCGGE are nonlocal except for $\hat{I}_{k}$.

In practice, it is a hard task both analytically and numerically to determine all the generalized temperatures for the exact $N$-body NCGGE for $N \geqslant 2$ since it is essentially a many-body problem. However, it is conceptually important: There exists a systematic construction of the GGE that is exact for all the less-than- $N$-body observables at finite system size. Below, we discuss some special NCGGEs of practical relevance: the exact one-body and approximate two-body NCGGEs.

\section{APPLICATION OF EXACT ONE-BODY NCGGE}

As shown above, the one-body NCGGE (11) exactly describes all the one-body observables unlike the conventional CGGE. We further study how this NCGGE works for twobody observables. Fortunately, we can analytically obtain the generalized temperatures $\lambda_{k}$ and $\omega_{k}$. Although we leave the detail in Appendix D, an important idea is to perform a unitary transformation in each $(k,-k)$ subspace: $\left(\hat{d}_{k}^{\dagger}, \hat{d}_{-k}^{\dagger}\right)=$ $\left(\hat{c}_{k}^{\dagger}, \hat{c}_{-k}^{\dagger}\right) U_{k}$, which diagonalizes the exponent in Eq. (11). Then we have a diagonal form

$$
\hat{\rho}_{1 \mathrm{NC}}=\frac{1}{Z_{1 \mathrm{NC}}} \prod_{k} \exp \left(-\eta_{k} \hat{I}_{k}^{d}\right),
$$

where $\hat{I}_{k}^{d}=\hat{d}_{k}^{\dagger} \hat{d}_{k}$ is the conserved quantity in the new basis and $\eta_{k}$ is some linear combination of $\lambda_{k}$ and $\omega_{k}$. Equation (14) is useful for obtaining the generalized temperatures (see Appendix D).

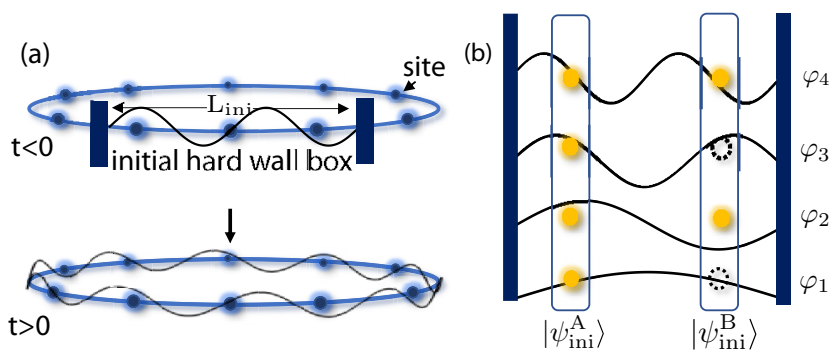

FIG. 1. (a) Schematic illustration of dynamics protocol. (b) Illustration of two initial states $\left|\psi_{\text {ini }}^{\mathrm{A}}\right\rangle$ and $\left|\psi_{\text {ini }}^{\mathrm{B}}\right\rangle$. Filled circles represent the occupied one-particle energy eigenstates.

To test the accuracy of $\hat{\rho}_{1 \mathrm{NC}}$, we consider a concrete initial state and its dynamics under the Hamiltonian (8). As shown in Fig. 1(a), we suppose an initial hard wall box, which confines $N$ particles to the sites $1 \leqslant i \leqslant L_{\text {ini }}\left(N \leqslant L_{\text {ini }}\right)$. The one-particle energy eigenstates within the box are $\varphi_{n}(j)=$ $\left(L_{\text {ini }}+1\right)^{-1 / 2} \sin \left[\pi n j /\left(L_{\text {ini }}+1\right)\right]$ as illustrated in Fig. 1(b). Introducing the creation operators for these eigenstates as $\Phi_{n}^{\dagger}=\sum_{j=1}^{L_{\text {ini }}} \varphi_{n}(j) \hat{c}_{j}^{\dagger}$, we consider the following two initial states: the ground state $\left|\psi_{\text {ini }}^{\mathrm{A}}\right\rangle=\prod_{n=1}^{N} \Phi_{n}^{\dagger}|0\rangle$ and an excited state $\left|\psi_{\text {ini }}^{\mathrm{B}}\right\rangle=\prod_{n=1}^{N} \Phi_{2 n}^{\dagger}|0\rangle$ (for $N \leqslant L_{\text {ini }} / 2$ ). We remove the hard wall instantaneously at time $t=0$, let these initial states evolve under $\hat{H}$, or freely expand into the entire $L$ sites, and analyze the long-time average of various observables.

Figure 2 displays the values of the additional conserved quantities $\left|\left\langle\hat{J}_{k}\right\rangle_{\text {ini }}\right| \equiv\left|\left\langle\psi_{\text {ini }}\left|\hat{J}_{k}\right| \psi_{\text {ini }}\right\rangle\right|$ for $\left|\psi_{\text {ini }}\right\rangle=\left|\psi_{\text {ini }}^{\mathrm{A}}\right\rangle$ and $\left|\psi_{\text {ini }}^{\mathrm{B}}\right\rangle$. While $\left|\left\langle\hat{J}_{k}\right\rangle_{\text {ini }}\right|$ are almost zero for most $k$ in $\left|\psi_{\text {ini }}^{\mathrm{A}}\right\rangle$, it has large values for $0<k<2 \pi / 3$ in $\left|\psi_{\text {ini }}^{\mathrm{B}}\right\rangle$. Thus $\hat{J}_{k}$ is less important for the GGE in case of $\left|\psi_{\text {ini }}^{\mathrm{A}}\right\rangle$ and the generalized temperatures for $\hat{J}_{k}$ are almost zero for most $k$. On the other hand, in case of $\left|\psi_{\text {ini }}^{\mathrm{B}}\right\rangle$, we should use $\hat{J}_{k}$ in the GGE and the generalized temperatures have large absolute values.

To compare the one-body NCGGE and the conventional CGGE, we consider some two-body observables since we have already shown that one-body observables are exactly described by the one-body NCGGE. To highlight the role of $\hat{J}_{k}$, we take $\left|\psi_{\mathrm{ini}}^{\mathrm{B}}\right\rangle$ as an initial state and focus on the



FIG. 2. The values of additional conserved quantities $\left|\left\langle\hat{J}_{k}\right\rangle_{\text {ini }}\right|$ in the initial states $\left|\psi_{\text {ini }}^{\mathrm{A}}\right\rangle$ and $\left|\psi_{\text {ini }}^{\mathrm{B}}\right\rangle$ with $L=600, L_{\text {ini }}=360$, and $N=$ 120. Results are not shown for $k<0$ since $\left|\left\langle\hat{J}_{k}\right\rangle_{\text {ini }}\right|=\left|\left\langle\hat{J}_{-k}\right\rangle_{\text {ini }}\right|$. 


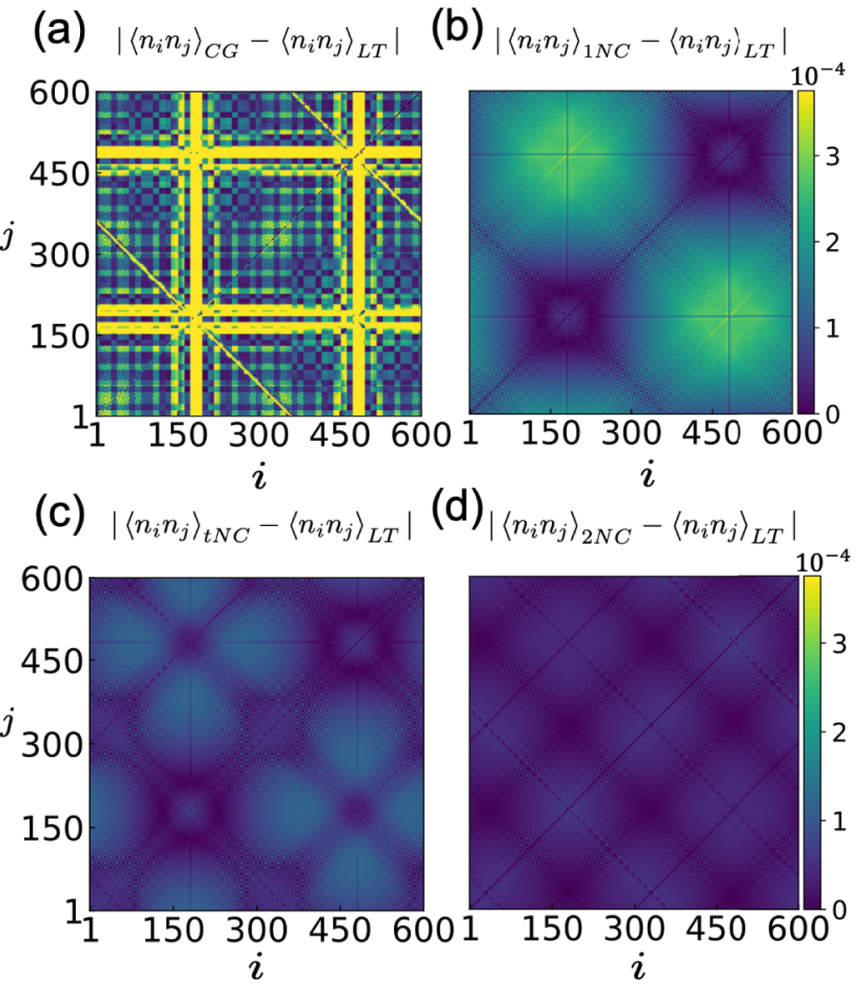

FIG. 3. Error of GGEs $\left|\left\langle\hat{n}_{i} \hat{n}_{j}\right\rangle_{\mathrm{GGE}}-\left\langle\hat{n}_{i} \hat{n}_{j}\right\rangle_{\mathrm{LT}}\right|$ for the densitydensity correlation between sites $i$ and $j$ calculated with the (a) CGGE, (b) one-body NCGGE, (c) trigonal NCGGE, and (d) twobody NCGGE with $L=600, L_{\mathrm{ini}}=360$, and $N=120$. In all panels, we use the initial state $\left|\psi_{\text {ini }}^{\mathrm{B}}\right\rangle$, and implicitly assume the normal ordering for $\hat{n}_{i} \hat{n}_{j}$ (see footnote ${ }^{1}$ ).

density-density correlation $\hat{n}_{i} \hat{n}_{j}\left(n_{i} \equiv \hat{c}_{i}^{\dagger} \hat{c}_{i}\right)^{1}$ and calculate the error of the GGEs $\left|\left\langle\hat{n}_{i} \hat{n}_{j}\right\rangle_{\mathrm{GGE}}-\left\langle\hat{n}_{i} \hat{n}_{j}\right\rangle_{\mathrm{LT}}\right|$, where GGE means the one-body NCGGE (1NC) or CGGE (C). We plot these errors in Figs. 3(a) and 3(b), finding $\hat{\rho}_{1 \mathrm{NC}}$ more accurate than the CGGE as a whole. We turn our attention further to local physical quantities $n_{j} n_{j+1}$, which are 1-local operators and the sub-diagonal components of Fig. 3. For a quantitative comparison of the local observables, we plot the expectation values of $\hat{n}_{j} \hat{n}_{j+1}$ in Fig. 4(a) and the errors of the GGEs $\left|\left\langle\hat{n}_{j} \hat{n}_{j+1}\right\rangle_{\mathrm{GGE}}-\left\langle\hat{n}_{j} \hat{n}_{j+1}\right\rangle_{\mathrm{LT}}\right|$ in Fig. 4(b). We find that $\hat{\rho}_{1 \mathrm{NC}}$ describes the long-time average $\left\langle\hat{n}_{j} \hat{n}_{j+1}\right\rangle_{\mathrm{LT}}$ better than the CGGE for most $j$ in Fig. 4 . It is noteworthy that the $\hat{\rho}_{1 \mathrm{NC}}$ captures the characteristic peaks of $\left\langle\hat{n}_{j} \hat{n}_{j+1}\right\rangle_{\mathrm{LT}}$ while the CGGE cannot. These characteristic peaks are related to the inversion symmetry and not present for $\left|\psi_{\text {ini }}^{\mathrm{A}}\right\rangle$ (see Appendix H), for which the improvement by $\hat{\rho}_{1 \mathrm{NC}}$.

We also examine how the errors scale in the system size $L$ with ratios $N / L_{\text {ini }}$ and $L_{\text {ini }} / L$ held fixed. We define the averaged error of the density-density correlation by $\Delta_{\text {ave }} \equiv \sum_{j}\left|\left\langle\hat{n}_{j} \hat{n}_{j+1}\right\rangle_{\mathrm{GGE}}-\left\langle\hat{n}_{j} \hat{n}_{j+1}\right\rangle_{\mathrm{LT}}\right| / L$, which is plotted for GGEs at several system sizes in Fig. 5(b). The error is much smaller for $\hat{\rho}_{1 \mathrm{NC}}$, and decreases as $\propto 1 / L$ to vanish in

${ }^{1}$ We actually calculate the normal-ordered operator $: \hat{n}_{i} \hat{n}_{j}:=$ $\hat{c}_{i}^{\dagger} \hat{c}_{j}^{\dagger} \hat{c}_{j} \hat{c}_{i}$ to remove the unwanted one-body contributions.



FIG. 4. (a) Expectation values of local density-density correlation of $\left\langle n_{j} n_{j+1}\right\rangle$ in the CGGE, one-body NCGGE, trigonal NCGGE, two-body NCGGE, and long-time average for the initial state $\left|\psi_{\text {ini }}^{\mathrm{B}}\right\rangle$ with $L=600, L_{\mathrm{ini}}=360$, and $N=120$. There are the characteristic peaks which cannot be captured by the CGGE at the high-symmetry points of the initial state $\left|\psi_{\text {ini }}^{\mathrm{B}}\right\rangle, j=L_{\text {ini }} / 2$ and $\left(L_{\text {ini }}+L\right) / 2$. At the high-symmetry points, the expectation value of the correlation function in long-time average and NCGGEs are zero, but the CGGE does not. (b) Error of GGEs $\left|\left\langle\hat{n}_{j} \hat{n}_{j+1}\right\rangle_{\mathrm{GGE}}-\left\langle\hat{n}_{j} \hat{n}_{j+1}\right\rangle_{\mathrm{LT}}\right|$ for the local density-density correlation between sites $j$ and $j+1$.

the thermodynamic limit for both GGEs. ${ }^{2}$ Thus the CGGE also becomes accurate in this limit on average. However, when we use a more strict definition for the error defined by $\Delta_{\max } \equiv \max _{j}\left|\left\langle\hat{n}_{i} \hat{n}_{j+1}\right\rangle_{\mathrm{GGE}}-\left\langle\hat{n}_{j} \hat{n}_{j+1}\right\rangle_{\mathrm{LT}}\right|$, we come to a different conclusion: The one-body NCGGE becomes accurate as $L \rightarrow \infty$ while the CGGE does not, as shown in Fig. 5(a). This is due to the characteristic peaks shown in Fig. 4 and the maximum error of the CGGE occurs at the high-symmetry points $j=L_{\text {ini }} / 2$ and $\left(L_{\text {ini }}+L\right) / 2$. For the other initial state $\left|\psi_{\text {ini }}^{\mathrm{A}}\right\rangle$, as $L$ increases, $\Delta_{\max }$ of the CGGE also decreases as $\propto 1 / L$ because there are no characteristic peaks, which cannot be captured by the CGGE. These results show that the one-body NCGGE improves the GGE prediction quantitatively as a whole, but some of the local correlations such

\footnotetext{
${ }^{2}$ This power-law decay is a feature of integrable models [25], and the error decays exponentially in nonintegrable models $[20,60]$
} 

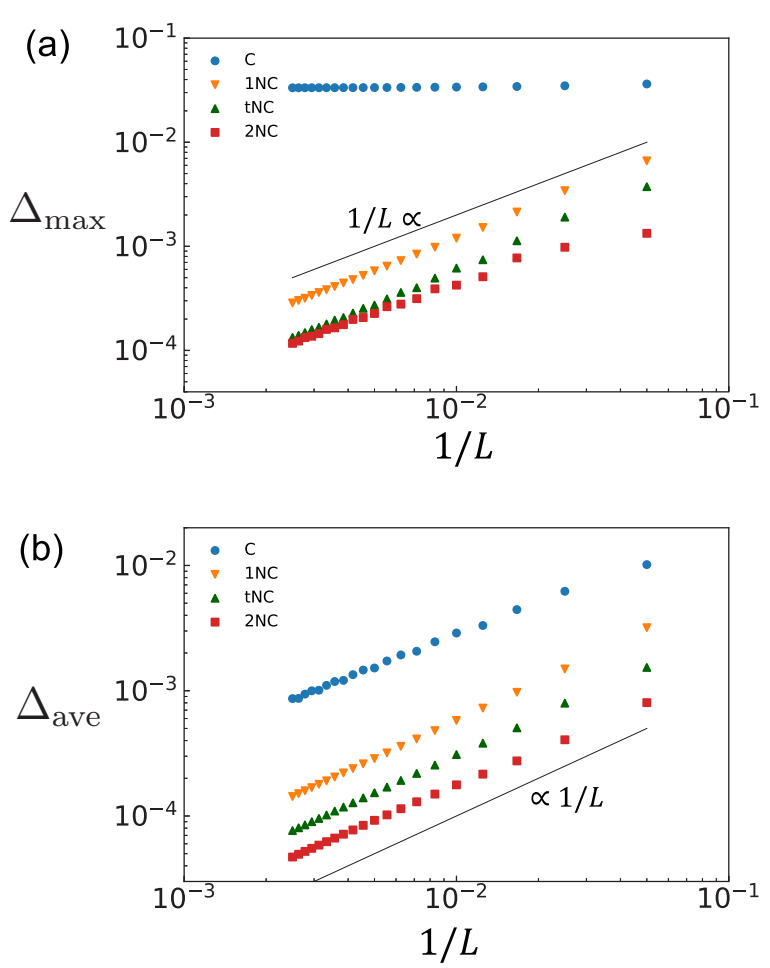

FIG. 5. The $L$ dependence of (a) maximum $\Delta_{\max }$ errors and (b) averaged $\Delta_{\text {ave }}$ errors of the expectation values of local densitydensity correlation of $\left\langle n_{j} n_{j+1}\right\rangle$ in the GGEs calculated with $L_{\text {ini }} / L=$ $3 / 5$ and $N / L_{\text {ini }}=1 / 3$ held fixed. The initial state is $\left|\psi_{\text {ini }}^{\mathrm{B}}\right\rangle$, and $L, L_{\text {ini }}$, and $N$ are all even at every data point.

as $n_{L_{\text {ini }} / 2} n_{L_{\text {ini }} / 2+1}$ can be improved by the one-body NCGGE qualitatively from the CGGE. The NCGGE can be necessary for accurately describing the actual stationary state even in the thermodynamic limit, depending on the initial state.

\section{IMPROVEMENT OF EXACT ONE-BODY NCGGE}

\section{A. Trigonal NCGGE}

Although it is difficult to implement the exact two-body NCGGE, we can partly include two-body conserved quantities, improving the one-body NCGGE. To inspect which conserved quantities are important, we calculate $\mid\left\langle\hat{I}_{k}^{d} \hat{I}_{q}^{d}\right\rangle_{\text {ini }}-$ $\left\langle\hat{I}_{k}^{d} \hat{I}_{q}^{d}\right\rangle_{1 N C} \mid$ and find that most deviations reside around the diagonal $(k=q)$ and antidiagonal $(k=-q)$ components (see Appendix G). Noting that $\left(\hat{I}_{k}^{d}\right)^{2}=\hat{I}_{k}^{d}$, we take the products of the adjacent pairs $\hat{I}_{k}^{d} \hat{I}_{k+\Delta k}^{d}$ with $\Delta k=2 \pi / L$, defining the following trigonal NCGGE:

$$
\hat{\rho}_{\mathrm{tNC}}=\frac{1}{Z_{\mathrm{tNC}}} \exp \left[-\sum_{k}\left(\tilde{\eta}_{k} \hat{I}_{k}^{d}+\Lambda_{k} \hat{I}_{k}^{d} \hat{I}_{k+\Delta k}^{d}\right)\right],
$$

where $Z_{\mathrm{tNC}}$ is defined by $\operatorname{Tr} \hat{\rho}_{\mathrm{tNC}}=1$. Remarkably, we can efficiently obtain the generalized temperatures $\tilde{\eta}_{k}$ and $\Lambda_{k}$ numerically by a method similar to the transfer matrix for the one-dimensional Ising model (see Appendix E).

The trigonal NCGGE thus implemented leads to a quantitative improvement of the one-body NCGGE. The error of the two-body conserved quantities $\hat{I}_{k}^{d} \hat{I}_{q}^{d}$ in both initial state $\left|\psi_{\mathrm{A}}\right\rangle$ and $\left|\psi_{\mathrm{B}}\right\rangle$ is reduced near the diagonal $(k=q)$ components (see Appendix G).

\section{B. Two-body NCGGE}

When we take all the two-body conserved quantities $\hat{I}_{k}^{d} \hat{I}_{q}^{d}$ into the GGE, the explicit calculation of the generalized temperatures is a very hard task. We call this ideal ensemble as the two-body NCGGE. We remark that this two-body NCGGE is different from the exact two-body NCGGE, which also involves two-body conserved quantities not in the form of $\hat{I}_{k}^{d} \hat{I}_{q}^{d}$.

Interestingly, without having the generalized temperatures, we can calculate the expectation value of the observables in the two-body NCGGE in the free fermion model from the information of the initial conditions. The density matrix of the two-body NCGGE is formally written as

$$
\hat{\rho}_{2 \mathrm{NC}}=\frac{1}{Z_{2 \mathrm{NC}}} \exp \left(-\sum_{k} \tilde{\eta}_{k} \hat{I}_{k}^{d}-\sum_{k>q} \Lambda_{k q} \hat{I}_{k}^{d} \hat{I}_{q}^{d}\right),
$$

where $Z_{2 \mathrm{NC}}$ is defined by $\operatorname{Tr} \hat{\rho}_{2 \mathrm{NC}}=1$. Let us consider a general two-body observable $\hat{A}^{(2)}=$ $\sum_{k_{1}, k_{2}, q_{1}, q_{2}} \tilde{A}_{k_{1} k_{2} ; q_{1} q_{2}} \hat{d}_{k_{1}}^{\dagger} \hat{d}_{k_{2}}^{\dagger} \hat{d}_{q_{2}} \hat{d}_{q_{1}}$ in the two-body NCGGE. In taking its expectation value for $\hat{\rho}_{2 \mathrm{NC}}$, only two kinds of contributions $k_{1}=q_{1}$ and $k_{2}=q_{2}$ or $k_{1}=q_{2}$ and $k_{2}=q_{1}$ are nonvanishing

$$
\begin{aligned}
\left\langle\hat{A}^{(2)}\right\rangle_{2 \mathrm{NC}} & =\sum_{k, q}\left(\tilde{A}_{k q ; k q}-\tilde{A}_{k q ; q k}\right)\left\langle\hat{I}_{k}^{d} \hat{I}_{q}^{d}\right\rangle_{2 \mathrm{NC}} \\
& =\sum_{k, q}\left(\tilde{A}_{k, q ; k q}-\tilde{A}_{k q ; q k}\right)\left\langle\hat{I}_{k}^{d} \hat{I}_{q}^{d}\right\rangle_{\mathrm{ini}} .
\end{aligned}
$$

To obtain the last equality, we have used the determining equations for the generalized temperatures, $\left\langle\hat{I}_{k}^{d} \hat{I}_{q}^{d}\right\rangle_{2 \mathrm{NC}}=\left\langle\hat{I}_{k}^{d} \hat{I}_{q}^{d}\right\rangle_{\text {ini }}$

We plot in Fig. 3 the error of the trigonal NCGGE (c) and the two-body NCGGE (d) for the density-density correlation $\hat{n}_{j} \hat{n}_{j+1}$, where the initial state is $\left|\psi_{\text {ini }}^{B}\right\rangle$. In Fig. 5, we observe qualitative features including $\Delta_{\text {ave }} \propto 1 / L$ similar to those of the one-body NCGGE. The more two-body nonlocal conserved quantities we take into the GGE, the more the GGE predictions of the nonlocal correlations are improved (which corresponds to the much-off-diagonal element in Fig. 3). We can see significant reductions of the errors $\Delta_{\text {ave }}$ and $\Delta_{\max }$ in Fig. 5 in both the trigonal NCGGE and the two-body NCGGE and the reductions are larger in the two-body NCGGE than in the trigonal NCGGE.

\section{SUMMARY AND OUTLOOK}

Introducing noncommutative sets of conserved quantities and the observable projection idea, we have systematically shown that the NCGGE describes the long-time behavior of isolated quantum systems better than the conventional CGGE. For noninteracting integrable systems, we have explicitly constructed the exact $N$-body NCGGE that describes the long-time average of up-to- $N$-body observables without an error even at finite system size. Besides, we have shown that the one-body NCGGE, the trigonal NCGGE, and the two-body NCGGE can be numerically implemented and describe two-body observables well. We note that the additional 
noncommutative conserved quantities are nonlocal. However, there exist local observables which need these nonlocal additional conserved quantities for qualitative description depending on the initial state.

The implementation of the NCGGE in interacting integrable systems is an important open problem. This problem is challenging because noncommutative sets of conserved quantities are not explored well in these systems and our approach presented for noninteracting systems does not apply. However, as we have remarked in Sec. III, one can numerically conduct the observable projection scheme for moderate system sizes and test whether the NCGGE is necessary or not. The numerical operator projection can be performed by the Gram-Schmidt orthogonalization and by the exact simultaneous diagonalization of the commutative local conserved quantities once we know the explicit form of the commutative local conserved quantities derived from the transfer matrix (see Appendix $\mathrm{C}$ for more details). With this analysis, we can see whether the NCGGE is needed or not in interacting integrable systems. Moreover, we may even find new conserved quantities that do not commute with the well-known commutative ones.

Finally, we remark that the quantum-information-theoretic thermodynamics using noncommutative conserved quantities has attracted attention [57]. An experimental protocol for its realization has been proposed in small nonintegrable systems [58]. Our NCGGE arising in large integrable systems provides another route to the quantum-information-theoretic thermodynamics.

\section{ACKNOWLEDGMENTS}

We thank H. Tsunetsugu for fruitful discussion and L. Piroli for his helpful comment on our manuscript. This work was supported by JSPS KAKENHI Grant No. JP18K13495. K.F. acknowledges support by the Forefront Physics and Mathematics Program to Drive Transformation (FoPM), WINGS Program, the University of Tokyo.

\section{APPENDIX A: THE UNIQUENESS OF THE GENERALIZED TEMPERATURE IN NCGGE}

We will show the generalized temperature of the NCGGE can be determined uniquely. In other words, we will show the equation $\left\langle\hat{Q}_{\alpha}\right\rangle_{\text {ini }}=\operatorname{Tr}\left(\hat{\rho}_{\mathrm{GGE}} \hat{Q}_{\alpha}\right)$ has a unique solution for $\left\{\lambda_{\alpha}\right\}$ if the conserved quantities are linearly independent. Let $\mathcal{S}$ denote the real linear space spanned by the linearly independent set of conserved quantities $\left\{\hat{Q}_{\alpha}\right\}$. All the elements of $\mathcal{S}$ are Hermitian conserved quantities. The exponent of the GGE $\hat{X} \equiv-\sum_{\alpha} \lambda_{\alpha} \hat{Q}_{\alpha}$ belongs to $\mathcal{S}$, and $\hat{\rho}_{\mathrm{GGE}}$ is written as $\hat{\rho}_{\mathrm{GGE}}=e^{\hat{X}} / \operatorname{Tr} e^{\hat{X}}$. Substituting $\hat{\rho}_{\mathrm{GGE}}=e^{\hat{X}} / \operatorname{Tr} e^{\hat{X}}$ into the entropy $\Psi$ in Eq. (4), we get

$$
\Phi(\hat{X}) \equiv \Psi\left(\hat{\rho}_{\mathrm{GGE}},\left\{\lambda_{\alpha}\right\}\right)=\ln \operatorname{Tr} e^{\hat{X}}-\langle\hat{X}\rangle_{\mathrm{ini}}
$$

The problem is reduced to the proof of the convexity of $\Phi(\hat{X})$ over $\mathcal{S}$, more specifically, the proof of the inequality $\Phi\left(\left(\hat{X}_{1}+\hat{X}_{2}\right) / 2\right) \leqslant\left(\Phi\left(\hat{X}_{1}\right)+\Phi\left(\hat{X}_{2}\right)\right) / 2$, where $\hat{X}_{1}, \hat{X}_{2} \in \mathcal{S}$ are arbitrary. The second terms of $\Phi$ are canceled, and what we should prove becomes

$$
\left(\operatorname{Tr} e^{\frac{1}{2}\left(\hat{X}_{1}+\hat{X}_{2}\right)}\right)^{2} \leqslant\left(\operatorname{Tr} e^{\hat{X}_{1}}\right)\left(\operatorname{Tr} e^{\hat{X}_{2}}\right) .
$$

When $\left\{\hat{Q}_{\alpha}\right\}$ is a commutative set, (A2) immediately holds by using the Cauchy-Schwarz inequality as discussed below in the noncommutative case.

When $\left\{\hat{Q}_{\alpha}\right\}$ is a noncommutative set, we can utilize the Golden-Thompson inequality [59] to the left-hand side of (A2) because $e^{\frac{1}{2} \hat{X}_{1}}$ and $e^{\frac{1}{2} \hat{X}_{2}}$ are positive semi-definite. Then we can see

$$
\operatorname{Tr} e^{\frac{1}{2}\left(\hat{X}_{1}+\hat{X}_{2}\right)} \leqslant \operatorname{Tr} e^{\frac{1}{2} \hat{X}_{1}} e^{\frac{1}{2} \hat{X}_{2}} .
$$

Calculating the trace of rhs of (A3) with an arbitrary basis $\{|i\rangle\}$ and using the Cauchy-Schwarz inequality, we find

$$
\begin{aligned}
\left(\operatorname{Tr} e^{\frac{1}{2} \hat{X}_{1}} e^{\frac{1}{2} \hat{X}_{2}}\right)^{2} & =\left(\sum_{i, j}\left\langle i\left|e^{\frac{1}{2} \hat{X}_{1}}\right| j\right\rangle\left\langle j\left|e^{\frac{1}{2} \hat{X}_{2}}\right| i\right\rangle\right)^{2} \\
& \leqslant\left(\sum_{i, j}\left|\left\langle i\left|e^{\frac{1}{2} \hat{X}_{1}}\right| j\right\rangle\right|^{2}\right)\left(\sum_{i, j}\left|\left\langle j\left|e^{\frac{1}{2} \hat{X}_{2}}\right| i\right\rangle\right|^{2}\right) \\
& (\because \text { Cauchy-Schwarz inequality) } \\
& =\left(\sum_{i}\left\langle i\left|e^{\hat{X}_{1}}\right| i\right\rangle\right)\left(\sum_{i}\left\langle i\left|e^{\hat{X}_{2}}\right| i\right\rangle\right) \\
& =\left(\operatorname{Tr} e^{\hat{X}_{1}}\right)\left(\operatorname{Tr} e^{\hat{X}_{2}}\right) .
\end{aligned}
$$

The equality condition of the inequality is $\hat{X}_{1} \propto \hat{X}_{2}$, which does not hold in the case that $\hat{X}_{1}$ and $\hat{X}_{2}$ do not commute. This completes the proof. Then we can see $\Phi(\hat{X})$ is convex over $\mathcal{S}$ and there is the unique minimum $\hat{X}^{*}$. Since $\left\{\hat{Q}_{\alpha}\right\}$ is independent each other, the coefficients of $\hat{X}^{*}$ are determined uniquely, and these coefficients are the unique solution of the generalized temperatures. Note that this proof is the natural extension of the commutative case.

\section{APPENDIX B: CONDITION FOR THE EXISTENCE OF THE ADDITIONAL LOCAL CONSERVED QUANTITIES}

We show a condition for the existence of the additional local conserved quantities which should be incorporated into the GGE. The definition of the complete set of the conserved quantities is the set of all the local or quasilocal conserved quantities.

The conserved quantity $\hat{Q}_{\alpha}$ is local when $\left\langle\hat{A}, \hat{Q}_{\alpha}\right\rangle^{2} /\left\langle\hat{Q}_{\alpha}, \hat{Q}_{\alpha}\right\rangle>0$ for some local observable $\hat{A}$. Here, $\hat{A}$ is traceless and normalized as $0<\lim _{L \rightarrow \infty}\|\hat{A}\|<\infty$ where $\|\hat{A}\| \equiv \sqrt{\langle\hat{A}, \hat{A}\rangle}$ and $L$ denotes the system size.

If the norm of $\hat{\bar{A}}_{\perp}$ does not vanish in the thermodynamic limit, i.e.,

$$
\lim _{L \rightarrow \infty}\left\|\hat{\bar{A}}_{\perp}\right\|^{2}>0
$$

then $\hat{\bar{A}}_{\perp}$ is an additional local conserved quantity which should be incorporated into the GGE. To see this, we first recall that $\hat{\bar{A}}_{\perp}$ is a conserved quantity. Then, the locality of 
$\hat{\bar{A}}_{\perp}$ follows from the identity

$$
\frac{\left\langle\hat{A}, \hat{\bar{A}}_{\perp}\right\rangle^{2}}{\left\langle\hat{\bar{A}}_{\perp}, \hat{\bar{A}}_{\perp}\right\rangle}=\left\langle\hat{\bar{A}}_{\perp}, \hat{\bar{A}}_{\perp}\right\rangle=\left\|\hat{\bar{A}}_{\perp}\right\|^{2}>0,
$$

where we have used $\left\langle\hat{\bar{A}}_{\perp}, \hat{\bar{A}}_{\perp}\right\rangle=\left\langle\hat{A}, \hat{\bar{A}}_{\perp}\right\rangle[56]$.

We remark that the opposite is not true (see Supplemental Material of Ref. [56]). Namely, $\lim _{L \rightarrow \infty}\left\|\hat{\bar{A}}_{\perp}\right\|=0$ does not necessarily mean the absence of the additional local conserved quantities and more over, $\lim _{L \rightarrow \infty} \hat{\bar{A}}_{\perp}=0$ as an operator.

\section{APPENDIX C: HOW TO DETECT THE NECESSITY OF NONCOMMUTATIVE CONSERVED QUANTITIES}

Now we discuss how to judge whether noncommutative conserved quantities need to be involved in the GGE or not when we have only commutative set of conserved quantities $\left\{\hat{Q}_{\alpha}^{\mathrm{C}}\right\}:\left[\hat{Q}_{\alpha}^{\mathrm{C}}, \hat{H}\right]=\left[\hat{Q}_{\alpha}^{\mathrm{C}}, \hat{Q}_{\beta}^{\mathrm{C}}\right]=0(\forall \alpha, \beta)$. Let $\hat{A}$ be the local observable of interest. We remark that $\left[\hat{\bar{A}}, \hat{Q}_{\alpha}^{\mathrm{C}}\right] \neq 0$ in general while $[\hat{\bar{A}}, \hat{H}]=0$ follows from the definition. From now on, we suppose that $\hat{Q}_{\alpha}^{\mathrm{C}}$, s are orthonormal and $\hat{H}$ is written as a linear combination of $\hat{Q}_{\alpha}^{\mathrm{C}}$ 's. In this situation, we can decompose $\hat{\bar{A}}$ into two parts:

$$
\hat{\bar{A}}=\hat{\bar{A}}^{\mathrm{C}}+\hat{\bar{A}}^{\mathrm{NC}}
$$

where $\hat{\bar{A}}^{\mathrm{C}}$ and $\hat{\bar{A}}^{\mathrm{NC}}$ does and does not commute with $\left\{\hat{Q}_{\alpha}^{\mathrm{C}}\right\}$, respectively. More explicitly, these are defined by $\hat{\bar{A}}^{\mathrm{C}} \equiv$ $\sum_{\left\{q_{\alpha}\right\}} \hat{P}_{\left\{q_{\alpha}\right\}} \hat{\bar{A}} \hat{P}_{\left\{q_{\alpha}\right\}}$ and $\hat{\bar{A}}^{\mathrm{NC}} \equiv \hat{\bar{A}}-\hat{\bar{A}}^{\mathrm{C}}$, where $\hat{P}_{\left\{q_{\alpha}\right\}}$ represents the projection operator onto the simultaneous eigenspace for $\left\{\hat{Q}_{\alpha}^{\mathrm{C}}\right\}$ in which $\hat{Q}_{\alpha}^{\mathrm{C}}$ has eigenvalue $q_{\alpha}$, respectively. Note that $\hat{\bar{A}}^{\mathrm{C}}$ and $\hat{\bar{A}}^{\mathrm{NC}}$ are orthogonal to each other $\left\langle\hat{\bar{A}}^{\mathrm{C}}, \hat{\bar{A}}^{\mathrm{NC}}\right\rangle=0$.

If $\hat{\vec{A}}^{\mathrm{NC}}$ does not vanish in the thermodynamic limit, then the CGGE can fail and the NCGGE is necessary as follows. Let us use the commutative orthogonal set of the conserved quantities $\left\{\hat{Q}_{\alpha}^{\mathrm{C}}\right\}$ for the operator projection. The diagonal component of the perpendicular term becomes $\hat{\bar{A}}_{\perp}=\hat{\bar{A}}_{\perp}^{\mathrm{C}}+\hat{\bar{A}}^{\mathrm{NC}}$ where $\hat{\bar{A}}_{\perp}^{\mathrm{C}}=\hat{\bar{A}}^{\mathrm{C}}-\sum_{\alpha} p_{A \alpha}^{\mathrm{C}} \hat{Q}_{\alpha}^{\mathrm{C}}$ and $p_{A \alpha}^{\mathrm{C}}=\left\langle p_{A \alpha}^{\mathrm{C}}, \hat{Q}_{\alpha}^{\mathrm{C}}\right\rangle /\left\langle\hat{Q}_{\alpha}^{\mathrm{C}}, \hat{Q}_{\alpha}^{\mathrm{C}}\right\rangle$ and the noncommutative part is unchanged.

Even if we do the best in the commutative part so that the commutative set $\left\{\hat{Q}_{\alpha}^{\mathrm{C}}\right\}$ is enough and $\lim _{L \rightarrow \infty} \hat{\bar{A}}_{\perp}^{\mathrm{C}}=0$, we still have the CGGE error as

$$
\lim _{L \rightarrow \infty}\left(\langle\hat{A}\rangle_{\mathrm{LT}}-\langle\hat{A}\rangle_{\mathrm{CGGE}}\right)=\lim _{L \rightarrow \infty}\left\langle\hat{\vec{A}}^{\mathrm{NC}}\right\rangle_{\mathrm{ini}},
$$

where $\langle\hat{A}\rangle_{\mathrm{CGGE}}$ is the CGGE expectation value of $\hat{A}$ and we use $\left\langle\hat{\vec{A}}^{\mathrm{NC}}\right\rangle_{\mathrm{CGGE}}=0$. Equation (C2) highlights the failure of the CGGE and the necessity of the NCGGE depending on the initial state.

\section{APPENDIX D: CALCULATION OF GENERALIZED TEMPERATURES FOR ONE-BODY NCGGE}

We study the explicit form of the generalized temperatures $\lambda_{k}$ and $\omega_{k}$. The density matrix of the one-body NCGGE is

$$
\hat{\rho}_{1 \mathrm{NC}}=\frac{1}{Z_{1 \mathrm{NC}}} \exp \left[-\sum_{k=-\pi}^{\pi}\left(\lambda_{k} \hat{I}_{k}+\omega_{k} \hat{J}_{k}\right)\right],
$$

where $Z_{1 \mathrm{NC}}=\operatorname{Tr} e^{-\sum_{k}\left(\lambda_{k} \hat{I}_{k}+\omega_{k} \hat{J}_{k}\right)}$. To make the density matrix Hermitian, we impose $\omega_{k}^{*}=\omega_{-k}$ because of $\hat{J}_{k}^{\dagger}=\hat{J}_{-k}$. We note that $\lambda_{k}$ is real since $\hat{I}_{k}^{\dagger}=\hat{I}_{k}$.

The generalized temperatures $\lambda_{k}$ and $\omega_{k}$ are uniquely and explicitly determined from the conditions $\left\langle\hat{I}_{k}\right\rangle_{\text {ini }}=\operatorname{Tr}\left[\hat{\rho}_{1 \mathrm{NC}} \hat{I}_{k}\right]$ and $\left\langle\hat{J}_{k}\right\rangle_{\text {ini }}=\operatorname{Tr}\left[\hat{\rho}_{1 \mathrm{NC}} \hat{J}_{k}\right]$ We note that $\hat{\rho}_{1 \mathrm{NC}}$ consists of product of the following $(k,-k)$-subspace operators:

$$
\begin{aligned}
\hat{X}_{k} & \equiv \lambda_{k} \hat{I}_{k}+\omega_{k} \hat{J}_{k}+\lambda_{-k} \hat{I}_{-k}+\omega_{-k} \hat{J}_{-k} \\
& =\left(\begin{array}{ll}
\hat{c}_{k}^{\dagger} & \hat{c}_{-k}^{\dagger}
\end{array}\right)\left(\begin{array}{cc}
\lambda_{k} & \omega_{k}^{*} \\
\omega_{k} & \lambda_{-k}
\end{array}\right)\left(\begin{array}{c}
\hat{c}_{k} \\
\hat{c}_{-k}
\end{array}\right) .
\end{aligned}
$$

Then the density matrix of the one-body NCGGE can be written as $\hat{\rho}_{1 \mathrm{NC}}=Z_{1 \mathrm{NC}}^{-1} \prod_{k} e^{-\hat{X}_{k}}$. We diagonalize the matrix in Eq. (D2). The Hermitian matrix can be written by the liner combination of Pauli matrices and identity matrix

$$
\begin{aligned}
\left(\begin{array}{cc}
\lambda_{k} & \omega_{k}^{*} \\
\omega_{k} & \lambda_{-k}
\end{array}\right) & =\bar{\lambda}_{k} I+\operatorname{Re} \omega_{k} \sigma_{x}+\operatorname{Im} \omega_{k} \sigma_{y}+\Delta \lambda_{k} \sigma_{z} \\
& =\bar{\lambda}_{k} I+\boldsymbol{a}_{k} \cdot \boldsymbol{\sigma}=\bar{\lambda}_{k} I+a_{k} \boldsymbol{n}_{k} \cdot \boldsymbol{\sigma},
\end{aligned}
$$

where $\quad \bar{\lambda}_{k}=\frac{1}{2}\left(\lambda_{k}+\lambda_{-k}\right), \quad \Delta \lambda_{k}=\frac{1}{2}\left(\lambda_{k}-\lambda_{-k}\right), \quad \boldsymbol{a}_{k}=$ $\left(\operatorname{Re} \omega_{k}, \operatorname{Im} \omega_{k}, \Delta \lambda_{k}\right)$, and $\sigma=\left(\sigma_{x}, \sigma_{y}, \sigma_{z}\right)$. We define the unit vector $\boldsymbol{n}_{k}=\boldsymbol{a}_{k} / a_{k}$, where $a_{k}=\left|\boldsymbol{a}_{k}\right|$.

We rotate $\boldsymbol{a}_{k} \cdot \boldsymbol{\sigma}$ to $\sigma_{z}$ by a unitary transformation

$$
U_{k}^{\dagger} \boldsymbol{n}_{k} \cdot \boldsymbol{\sigma} U_{k}=\sigma_{z} \text {. }
$$

Thus we obtain

$$
U_{k}^{\dagger}\left(\begin{array}{cc}
\lambda_{k} & \omega_{k}^{*} \\
\omega_{k} & \lambda_{-k}
\end{array}\right) U_{k}=\left(\begin{array}{cc}
\bar{\lambda}_{k}+a_{k} & 0 \\
0 & \bar{\lambda}_{k}-a_{k}
\end{array}\right) .
$$

An explicit form of the unitary transformation is given by

$$
\begin{aligned}
U_{k}^{\dagger} & =e^{i \frac{\phi_{k}}{2} \sigma_{z}} e^{i \frac{\theta_{k}}{2} \sigma_{y}} \\
& =\left(\begin{array}{cc}
e^{\frac{i}{2} \phi} \cos (\theta / 2) & e^{-\frac{i}{2} \phi} \sin (\theta / 2) \\
-e^{\frac{i}{2} \phi} \sin (\theta / 2) & e^{-\frac{i}{2} \phi} \cos (\theta / 2)
\end{array}\right),
\end{aligned}
$$

where $\theta_{k}$ and $\phi_{k}$ are the polar and azimuthal angles of $\boldsymbol{n}_{k}$. The corresponding transformation of the annihilation operators is

$$
\left(\begin{array}{c}
d_{k} \\
d_{-k}
\end{array}\right)=U_{k}^{\dagger}\left(\begin{array}{c}
c_{k} \\
c_{-k}
\end{array}\right) .
$$

Note that the unitary transformation preserves the anticommutation relations

$$
\left\{d_{\sigma k}, d_{\rho k}^{\dagger}\right\}=\left(U_{k}^{\dagger} U_{k}\right)_{\sigma \rho}=\delta_{\sigma \rho},
$$

where $\sigma$ and $\rho= \pm 1$. Then, $\hat{X}_{k}$ becomes

$$
\begin{aligned}
\hat{X}_{k} & =\left(\bar{\lambda}_{k}+a_{k}\right) d_{k}^{\dagger} d_{k}+\left(\bar{\lambda}_{k}-a_{k}\right) d_{-k}^{\dagger} d_{-k} \\
& =\eta_{k} \hat{I}_{k}^{d}+\eta_{-k} \hat{I}_{-k}^{d},
\end{aligned}
$$


where $\hat{I}_{k}^{d}=d_{k}^{\dagger} d_{k}$ is the rotated conserved quantitiy and $\eta_{ \pm k}=$ $\bar{\lambda}_{k} \pm a_{k}$. The density matrix is then diagonalized in the $d_{k}$ basis as

$$
\hat{\rho}_{1 \mathrm{NC}}=\frac{1}{Z_{1 \mathrm{NC}}} \prod_{k=-\pi}^{\pi} \exp \left(-\eta_{k} \hat{I}_{k}^{d}\right) .
$$

Note that $\hat{I}_{k}^{d}$ commutes with each other $\left[\hat{I}_{k}^{d}, \hat{I}_{q}^{d}\right]=0$, and $\lambda_{k}$ and $\omega_{k}$ are written as

$$
\begin{aligned}
& \lambda_{ \pm k}=\bar{\eta}_{k} \pm \Delta \eta_{k} \cos \theta_{k}, \\
& \omega_{ \pm k}=\Delta \eta_{k} e^{ \pm i \phi_{k}} \sin \theta_{k},
\end{aligned}
$$

where $\bar{\eta}_{k}=\left(\eta_{k}+\eta_{-k}\right) / 2$ and $\Delta \eta_{k}=\left(\eta_{k}-\eta_{-k}\right) / 2$. The determining equations for $\theta_{k}, \phi_{k}$, and $\eta_{ \pm k}$ are

$$
\begin{gathered}
\left\langle\hat{I}_{ \pm k}\right\rangle_{\text {ini }}=\frac{\cos ^{2} \theta_{k} / 2}{1+e^{\eta_{k}}}+\frac{\sin ^{2} \theta_{k} / 2}{1+e^{\eta_{-k}}}, \\
\left\langle\hat{J}_{ \pm k}\right\rangle_{\text {ini }}=\frac{e^{\mp i \phi_{k}}}{2} \sin \theta_{k}\left(\frac{1}{1+e^{\eta_{k}}}-\frac{1}{1+e^{\eta_{-k}}}\right) .
\end{gathered}
$$

Solving these equations, we have

$$
\begin{aligned}
& e^{i \phi_{k}}=-\left|\left\langle\hat{J}_{k}\right\rangle_{\mathrm{ini}}\right| /\left\langle\hat{J}_{k}\right\rangle_{\mathrm{ini}}, \\
& \cos \theta_{k}=-\frac{\left\langle\hat{I}_{k}\right\rangle_{\mathrm{ini}}-\left\langle\hat{I}_{-k}\right\rangle_{\mathrm{ini}}}{\sqrt{\left(\left\langle\hat{I}_{k}\right\rangle_{\mathrm{ini}}-\left\langle\hat{I}_{-k}\right\rangle_{\mathrm{ini}}\right)^{2}+4\left|\left\langle\hat{J}_{k}\right\rangle_{\mathrm{ini}}\right|^{2}}}, \\
& \sin \theta_{k}= \frac{2\left|\left\langle\hat{J}_{k}\right\rangle_{\mathrm{ini}}\right|}{\sqrt{\left(\left\langle\hat{I}_{k}\right\rangle_{\mathrm{ini}}-\left\langle\hat{I}_{-k}\right\rangle_{\mathrm{ini}}\right)^{2}+4\left|\left\langle\hat{J}_{k}\right\rangle_{\mathrm{ini}}\right|^{2}}}, \\
&\left\langle\hat{I}_{ \pm k}^{d}\right\rangle_{\mathrm{ini}}= \frac{1}{1+e^{\eta_{ \pm k}}} \\
&= \frac{\left\langle\hat{I}_{k}\right\rangle_{\mathrm{ini}}+\left\langle\hat{I}_{-k}\right\rangle_{\mathrm{ini}}}{2} \\
& \mp \sqrt{\left(\left\langle\hat{I}_{k}\right\rangle_{\mathrm{ini}}-\left\langle\hat{I}_{-k}\right\rangle_{\mathrm{ini}}\right)^{2} / 4+\left|\left\langle\hat{J}_{k}\right\rangle_{\mathrm{ini}}\right|^{2}} .
\end{aligned}
$$

Using Eqs. (D17)-(D20), we obtain the explicit forms of $\phi_{k}, \theta_{k}$, and $\eta_{k}$ as

$$
\begin{gathered}
\phi_{k}=\pi-\arg \left\langle\hat{J}_{k}\right\rangle_{\mathrm{ini}}, \\
\theta_{k}=-\tan ^{-1}\left[2\left|\left\langle\hat{J}_{k}\right\rangle_{\mathrm{ini}}\right| /\left(\left\langle\hat{I}_{k}\right\rangle_{\mathrm{ini}}-\left\langle\hat{I}_{-k}\right\rangle_{\mathrm{ini}}\right)\right], \\
\eta_{k}=\ln \left(\frac{1}{\left\langle\hat{I}_{k}^{d}\right\rangle_{\text {ini }}}-1\right) .
\end{gathered}
$$

Then, we obtain the generalized temperatures $\lambda_{k}$ and $\omega_{k}$ from Eqs. (D13) and (D14).

\section{APPENDIX E: DETERMINATION OF GENERALIZED TEMPERATURE FOR TRIGONAL NCGGE}

We discuss the generalized temperatures of the trigonal NCGGE. For this purpose in this section, we introduce an abuse of notation $\hat{I}_{K}^{d}$ for $\hat{I}_{k}^{d}$, where $K$ is an integer satisfying

$$
k=2 \pi K / L \quad \bmod 2 \pi .
$$

Then the density matrix of the trigonal NCGGE is

$$
\begin{aligned}
\hat{\rho}_{\mathrm{tNC}} & =\frac{1}{Z_{\mathrm{tNC}}} \exp \left(-\sum_{K} \tilde{\eta}_{K} \hat{I}_{K}^{d}-\sum_{K} \Lambda_{K} \hat{I}_{K}^{d} \hat{I}_{K+1}^{d}\right) \\
& =\frac{1}{Z_{\mathrm{tNC}}} \prod_{K=0}^{L-1} T_{K}\left(\hat{I}_{K}^{d}, \hat{I}_{K+1}^{d}\right),
\end{aligned}
$$

where $T_{K}\left(\hat{I}_{K}^{d}, \hat{I}_{K+1}^{d}\right)$ is the transfer matrix operator

$$
\begin{aligned}
& T_{K}\left(\hat{I}_{K}^{d}, \hat{I}_{K+1}^{d}\right) \\
& \quad=\exp \left[-\left(\Lambda_{K} \hat{I}_{K}^{d} \hat{I}_{K+1}^{d}+\left(\tilde{\eta}_{K} \hat{I}_{K}^{d}+\tilde{\eta}_{K+1} \hat{I}_{K+1}^{d}\right)\right) / 2\right] .
\end{aligned}
$$

In analogy with the Ising model in one dimension, we define the transfer matrix as

$$
\begin{aligned}
T_{K} & =\left(\begin{array}{cc}
T_{K}(1,1) & T_{K}(1,0) \\
T_{K}(0,1) & T_{K}(0,0)
\end{array}\right) \\
& =\left(\begin{array}{cc}
e^{-\Lambda_{K}-\left(\tilde{\eta}_{K}+\tilde{\eta}_{K+1}\right) / 2} & e^{-\tilde{\eta}_{K} / 2} \\
e^{-\tilde{\eta}_{K+1} / 2} & 1
\end{array}\right) .
\end{aligned}
$$

By using the transfer matrix, we can calculate the partition function and the expectation values of each conserved quantity in the trigonal NCGGE as

$$
\begin{aligned}
Z_{\mathrm{tNC}} & =\left(T_{0} T_{1} \ldots T_{L-1}\right)_{00}+\left(T_{0} T_{1} \ldots T_{L-1}\right)_{11}, \\
\left\langle\hat{I}_{K}^{d}\right\rangle_{\mathrm{tNC}} & =\frac{\left(T_{K} T_{K+1} \ldots T_{L-1} T_{0} T_{1} \ldots T_{K-1}\right)_{11}}{Z_{\mathrm{tNC}}}, \\
\left\langle\hat{I}_{K}^{d} \hat{I}_{K+1}^{d}\right\rangle_{\mathrm{tNC}} & =\frac{\left(T_{K}\right)_{11}\left(T_{K+1} \ldots T_{L-1} T_{0} T_{1} \ldots T_{K-1}\right)_{11}}{Z_{\mathrm{tNC}}} .
\end{aligned}
$$

We remark that the right-hand sides of these equations can be numerically evaluated in polynomial times rather than exponential ones.

The determining equations for the generalized temperatures are $\left\langle\hat{I}_{K}^{d}\right\rangle_{\mathrm{tNC}}=\left\langle\hat{I}_{K}^{d}\right\rangle_{\mathrm{ini}}$ and $\left\langle\hat{I}_{K}^{d} \hat{I}_{K+1}^{d}\right\rangle_{\mathrm{tNC}}=\left\langle\hat{I}_{K}^{d} \hat{I}_{K+1}^{d}\right\rangle_{\mathrm{ini}}$, which are equivalent to the following self-consistent equations for $T_{K}$ :

$$
\begin{aligned}
\left(T_{K}\right)_{11} & =\frac{Z_{\mathrm{tNC}}\left\langle\hat{I}_{K}^{d} \hat{I}_{K+1}^{d}\right\rangle_{\mathrm{ini}}}{\left(T_{K+1} \ldots T_{L-1} T_{0} \ldots T_{K-1}\right)_{11}}, \\
\left(T_{K}\right)_{10}= & \frac{Z_{t N C}\left(\left\langle\hat{I}_{K}^{d}\right\rangle_{\mathrm{ini}}-\left\langle\hat{I}_{K}^{d} \hat{I}_{K+1}^{d}\right\rangle_{\mathrm{ini}}\right)}{\left(T_{K+1} \ldots T_{L-1} T_{0} \ldots T_{K-1}\right)_{01}} .
\end{aligned}
$$

By iteratively calculating $T_{K}$, we obtain the generalized temperatures $\tilde{\eta}_{k}$ and $\Lambda_{k}$.

\section{APPENDIX F: $(k,-k)$-SUBSPACE NCGGE}

Although we cannot easily take two-body operator into the GGE, $\hat{I}_{k} \hat{I}_{-k}$ can be easily taken into the GGE because we can diagonalize the density matrix in each $(k,-k)$ subspace as the one-body NCGGE. However, when we use the initial state of the product of the single-particle state, the result is the same as the one-body NCGGE.

Note that $\hat{I}_{k} \hat{I}_{-k}$ is invariant under the unitary transformation, or $\hat{I}_{k} \hat{I}_{-k}=\hat{I}_{k}^{d} \hat{I}_{-k}^{d}$. We call the GGE with the conserved quantities $\hat{I}_{k}, \quad \hat{J}_{k}$ and $\hat{I}_{k} \hat{I}_{-k}$ as the $(k,-k)$ subspace GGE (sGGE). The density matrix of the sGGE is $\hat{\rho}_{\mathrm{sNC}}=\frac{1}{Z_{\mathrm{sNC}}} e^{-\sum_{0<k<\pi} \hat{Y}_{k}}$, where 
$Z_{\mathrm{sNC}}=\operatorname{Tr} e^{-\sum_{0<k<\pi} \hat{r}_{k}}$ is the partition function and

$$
\hat{Y}_{k}=\lambda_{k} \hat{I}_{k}+\omega_{k} \hat{J}_{k}+\lambda_{-k} \hat{I}_{-k}+\omega_{-k} \hat{J}_{-k}+\Gamma_{k} \hat{I}_{k} \hat{I}_{-k} \text {. }
$$

We rotate the basis as in the one-body NCGGE. The rotated form of $\hat{Y}_{k}$ by $U_{k}$ is

$$
\hat{Y}_{k}=\eta_{k} \hat{I}_{k}^{d}+\eta_{-k} \hat{I}_{-k}^{d}+\Gamma_{k} \hat{I}_{k}^{d} \hat{I}_{-k}^{d} .
$$

The definitions of these symbols are the same as the one-body NCGGE case. The initial state expectation values of the conserved quantities are

$$
\begin{gathered}
\left\langle\hat{I}_{ \pm k}\right\rangle_{\text {ini }}=\cos ^{2} \frac{\theta_{k}}{2} x_{ \pm k}+\sin ^{2} \frac{\theta_{k}}{2} x_{\mp k}+y_{k}, \\
\left\langle\hat{J}_{ \pm k}\right\rangle_{\text {ini }}=\frac{e^{\mp i \phi_{k}}}{2} \sin \theta_{k}\left(x_{k}-x_{-k}\right), \\
\left\langle\hat{I}_{k} \hat{I}_{-k}\right\rangle_{\text {ini }}=y_{k},
\end{gathered}
$$

where $x_{k}=e^{-\eta_{k}} / z_{k}, \quad y_{k}=e^{-\left(\eta_{k}+\eta_{-k}+\Gamma_{k}\right)} / z_{k} \quad$ and $\quad z_{k}=1+$ $e^{-\eta_{k}}+e^{-\eta_{-k}}+e^{-\left(\eta_{k}+\eta_{-k}+\Gamma_{k}\right)}$. Solving these equations for $x_{ \pm k}$ and $z_{k}$, we have

$$
\begin{aligned}
x_{ \pm k}= & \frac{\left\langle\hat{I}_{k}\right\rangle_{\text {ini }}+\left\langle\hat{I}_{-k}\right\rangle_{\text {ini }}}{2}-\left\langle\hat{I}_{k} \hat{I}_{-k}\right\rangle_{\text {ini }} \\
& \pm \sqrt{\left(\left\langle\hat{I}_{k}\right\rangle_{\text {ini }}-\left\langle\hat{I}_{-k}\right\rangle_{\text {ini }}\right)^{2} / 4+\left|\left\langle\hat{J}_{k}\right\rangle_{\text {ini }}\right|^{2}}, \\
z_{k} & =\frac{1}{1+\left\langle\hat{I}_{k} \hat{I}_{-k}\right\rangle_{\text {ini }}-\left\langle\hat{I}_{k}\right\rangle_{\text {ini }}-\left\langle\hat{I}_{k}\right\rangle_{\text {ini }}} .
\end{aligned}
$$

From these, we can see that the rotation angles $\phi_{k}$ and $\theta_{k}$ are the same as the one-body NCGGE (D21) and (D22), and $\left\langle\hat{I}_{ \pm k}^{d}\right\rangle_{\text {ini }}$ is also the same as (D20). We can calculate $\eta_{k}$ as $\eta_{k}=-\ln \left(x_{k} z_{k}\right)$. Therefore we can calculate the generalized temperatures $\lambda_{k}$ and $\omega_{k}$ with Eqs. (D13), (D14), (D21), and (D22). In addition, $\Gamma_{k}$ is obtained as

$$
\Gamma_{k}=\ln \frac{x_{k} x_{-k} z_{k}}{y_{k}} .
$$

We can calculate the explicit formula of the generalized temperatures of the $(k,-k)$ subspace NCGGE because $\hat{I}_{k}^{d} \hat{I}_{-k}^{d}$ is the operator which acts on the $(k,-k)$ subspace. The value of $\theta_{k}$ and $\phi_{k}$ are not affected whether $\hat{I}_{k}^{d} \hat{I}_{-k}^{d}$ is used in the GGE or not. Note that $\hat{I}_{k}^{d} \hat{I}_{-k}^{d}$ is invariant under the unitary transformation, i.e., $\hat{I}_{k}^{d} \hat{I}_{-k}^{d}=\hat{I}_{k} \hat{I}_{-k}$.

When the initial state is the product of the singleparticle state, there is no improvement in the $(k,-k)$ subspace NCGGE from the one-body NCGGE. This is because $\left\langle\hat{I}_{k}^{d} \hat{I}_{-k}^{d}\right\rangle_{1 \mathrm{NC}}=\left\langle\hat{I}_{k}\right\rangle_{\text {ini }}\left\langle\hat{I}_{-k}\right\rangle_{\text {ini }}-\left|\left\langle\hat{J}_{k}\right\rangle_{\text {ini }}\right|^{2}$ and $\left\langle\hat{I}_{k}^{d} \hat{I}_{-k}^{d}\right\rangle_{\mathrm{sNC}}=\left\langle\hat{I}_{k} \hat{I}_{-k}\right\rangle_{\mathrm{ini}}$ and we can easily show

$$
\left\langle\hat{I}_{k} \hat{I}_{-k}\right\rangle_{\text {ini }}=\left\langle\hat{I}_{k}\right\rangle_{\text {ini }}\left\langle\hat{I}_{-k}\right\rangle_{\text {ini }}-\left|\left\langle\hat{J}_{k}\right\rangle_{\text {ini }}\right|^{2},
$$

when the initial state is the product of the single-particle state. From this, we can see that the expectation values of the conserved quantities $\hat{I}_{k}^{d} \hat{I}_{-k}^{d}$ are the same in the one-body NCGGE and the $(k,-k)$ subspace NCGGE when the initial state is the product of the single-particle state. The difference of the fitting of the conserved quantities in the two NCGGE is only the fitting of the $\hat{I}_{k}^{d} \hat{I}_{-k}^{d}$. Therefore the expectation value of any observables in the one-body NCGGE and the $(k,-k)$ subspace NCGGE is the same when the initial state is the product of the single-particle state.
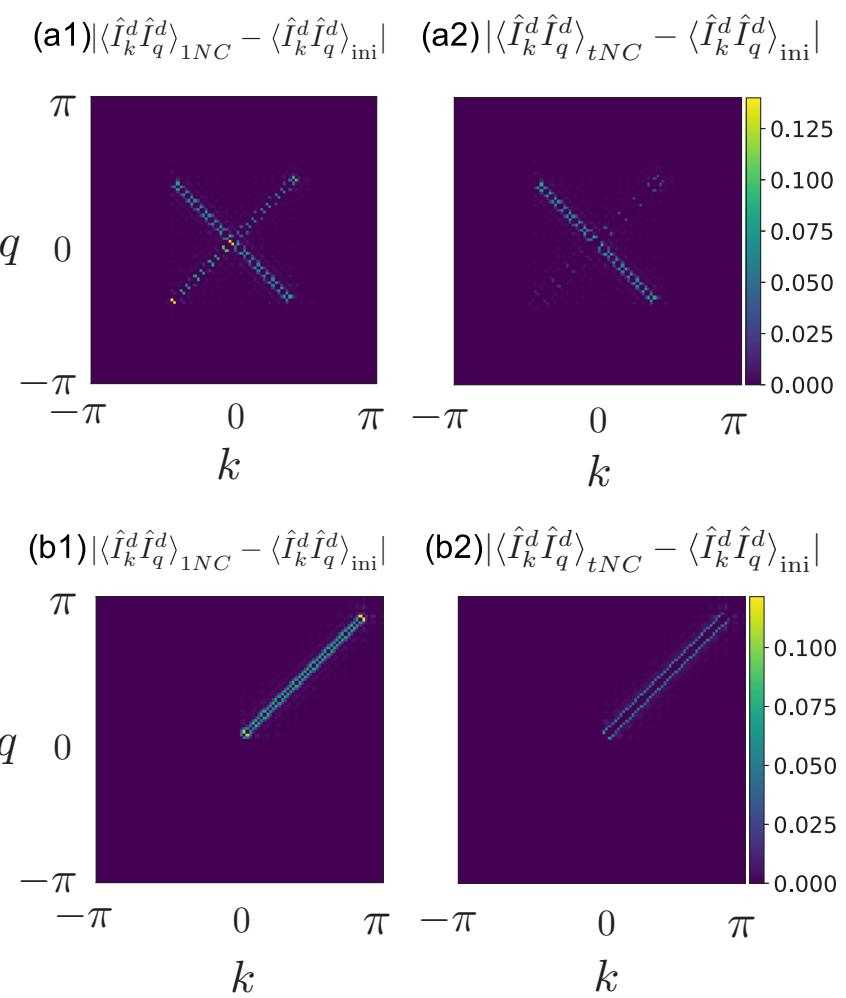

FIG. 6. The differences of the expectation value of $\hat{I}_{k}^{d} \hat{I}_{q}^{d}$ from the initial state expectation value are plotted. The one-body NCGGE case $\left|\left\langle\hat{I}_{k}^{d} \hat{I}_{q}^{d}\right\rangle_{\text {ini }}-\left\langle\hat{I}_{k}^{d} \hat{I}_{q}^{d}\right\rangle_{1 N C}\right|$ with the initial state $\left|\psi_{\text {ini }}^{A}\right\rangle$ is (a1) and that with the initial state $\left|\psi_{\text {ini }}^{B}\right\rangle$ is (b1). The trigonal NCGGE case $\left|\left\langle\hat{I}_{k}^{d} \hat{I}_{q}^{d}\right\rangle_{\text {ini }}-\left\langle\hat{I}_{k}^{d} \hat{I}_{q}^{d}\right\rangle_{t N C}\right|$ with the initial state $\left|\psi_{\text {ini }}^{A}\right\rangle$ is (a2) and that with the initial state $\left|\psi_{\text {ini }}^{B}\right\rangle$ is (b2). The color bars are common in upper panels and in lower panels respectivelly. The system size is $L=100$ and the particle number is $N=30$. The initial hard wall box is the size of $L_{\text {ini }}=70$.

The initial state used in this paper is the product of the single-particle state. Thus we do not use the $(k,-k)$ subspace NCGGE because the result is the same in the one-body NCGGE.

\section{APPENDIX G: FITTING OF TWO-BODY CONSERVED QUANTITIES IN ONE-BODY AND TRIGONAL NCGGEs}

We study how much the two-body conserved quantities $\hat{I}_{k}^{d} \hat{I}_{q}^{d}$ are fit by the one-body NCGGE or trigonal NCGGE. We plot $\left|\left\langle\hat{I}_{k}^{d} \hat{I}_{q}^{d}\right\rangle_{\text {ini }}-\left\langle\hat{I}_{k}^{d} \hat{I}_{q}^{d}\right\rangle_{1 \mathrm{NC}}\right|$ with the initial state $\left|\psi_{\mathrm{ini}}^{A}\right\rangle$ in Fig. 6(a1) and with the initial state $\left|\psi_{\text {ini }}^{B}\right\rangle$ in Fig. 6(b1). We also plot $\left|\left\langle\hat{I}_{k}^{d} \hat{I}_{q}^{d}\right\rangle_{\text {ini }}-\left\langle\hat{I}_{k}^{d} \hat{I}_{q}^{d}\right\rangle_{\mathrm{tNC}}\right|$ with the initial state $\left|\psi_{\mathrm{ini}}^{A}\right\rangle$ in Fig. 6(a2) and with the initial state $\left|\psi_{\text {ini }}^{B}\right\rangle$ in Fig. 6(b2). In both initial state case, We find that most deviations reside around the diagonal $(k=q)$ and antidiagonal $(k=-q)$ components in one-body NCGGE case. The trigonal NCGGE is also made from the two-body conserved quantities $\hat{I}_{k}^{d} \hat{I}_{k+\Delta k}^{d}$. Comparing Figs. 6(a1), 6(b1) and Figs. 6(a2) and 6(b2), we can see the fitting of the trigonal components of the two-body conserved quantities are improved from the one-body NCGGE. 


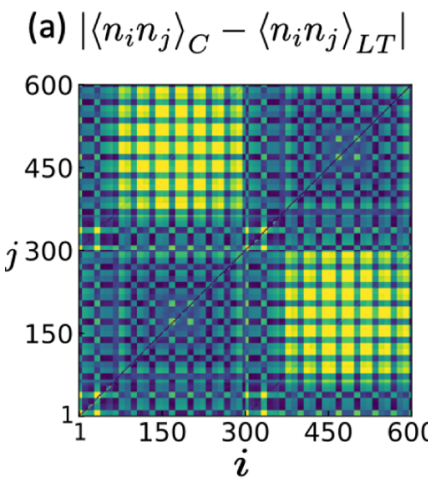

(b) $\left|\left\langle n_{i} n_{j}\right\rangle_{1 N C}-\left\langle n_{i} n_{j}\right\rangle_{L T}\right|$

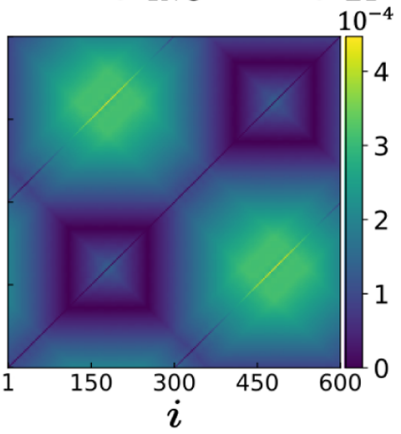

(c) $\left|\left\langle n_{i} n_{j}\right\rangle_{t N C}-\left\langle n_{i} n_{j}\right\rangle_{L T}\right|$
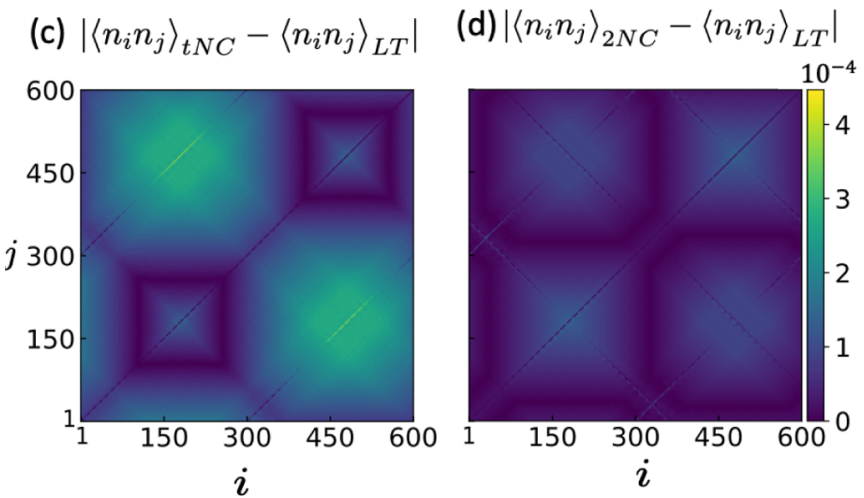

FIG. 7. Error of GGEs $\left|\left\langle\hat{n}_{i} \hat{n}_{j}\right\rangle_{\mathrm{GGE}}-\left\langle\hat{n}_{i} \hat{n}_{j}\right\rangle_{\mathrm{LT}}\right|$ for the densitydensity correlation between sites $i$ and $j$ calculated with the (a) CGGE, (b) one-body NCGGE, (c) trigonal NCGGE, (d) two-body NCGGE. In all panels, $L=600, L_{\text {ini }}=360$, and $N=120$. and we use the initial state $\left|\psi_{\text {ini }}^{\mathrm{A}}\right\rangle$, and implicitly assume the normal ordering for $\hat{n}_{i} \hat{n}_{j}$ (see footnote ${ }^{1}$ ).

\section{APPENDIX H: DENSITY-DENSITY CORRELATION FOR GROUND INITIAL STATE $\left|\psi_{\text {ini }}^{\mathrm{A}}\right\rangle$}

We show the expectation values of the density-density correlation in the CGGE and the NCGGEs for the ground initial state $\left|\psi_{\text {ini }}^{\mathrm{A}}\right\rangle$ in Fig. 7. We evaluate the error $\mid\left\langle\hat{n}_{i} \hat{n}_{j}\right\rangle_{\mathrm{GGE}}-$ $\left\langle\hat{n}_{i} \hat{n}_{j}\right\rangle_{\mathrm{LT}} \mid$, where GGE means the C-, one-body NC-, trigonal NC-, and two-body NC-GGE. We find that the more conserved quantities are used, the more accurate the GGE becomes. For a quantitative comparison of local observables, we plot the expectation values of $\hat{n}_{j} \hat{n}_{j+1}$ in Fig. 8(a). There are no
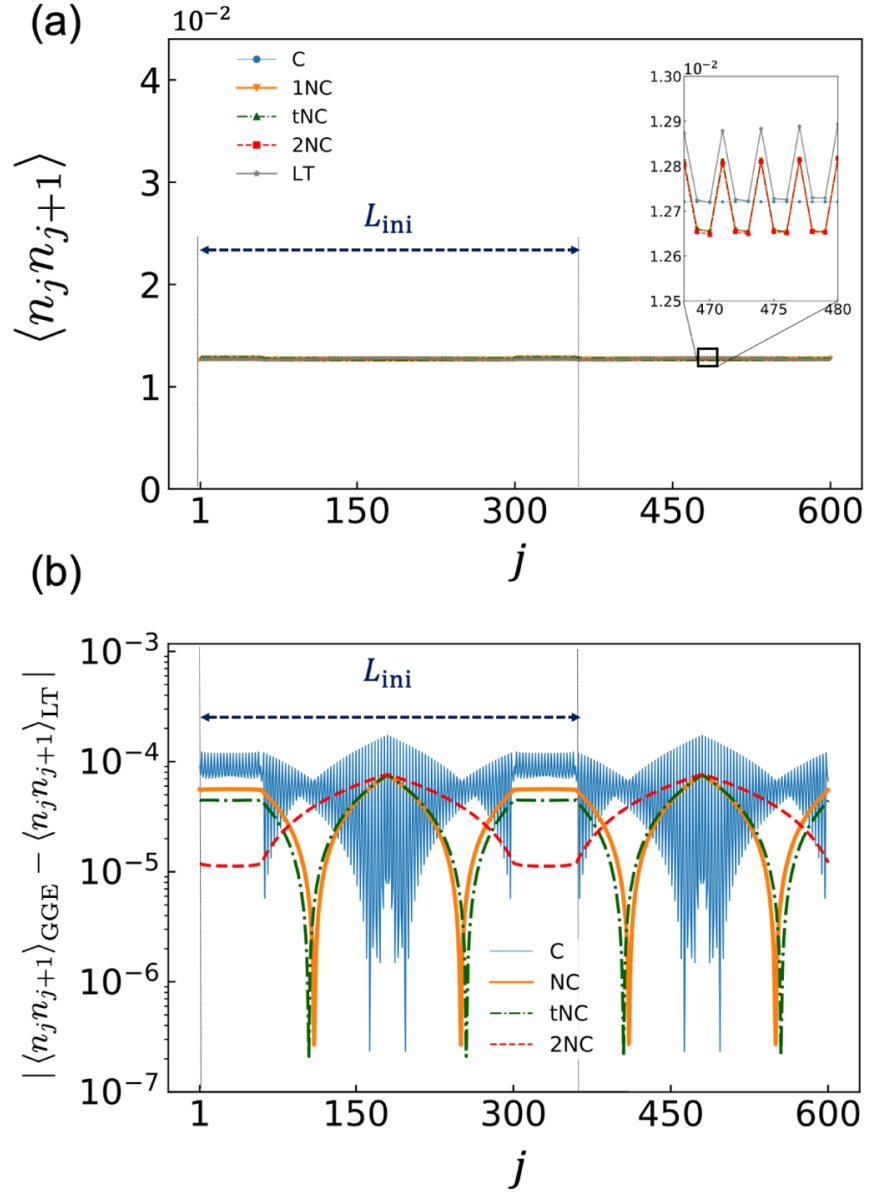

FIG. 8. (a) Expectation values of local density-density correlation of $\left\langle n_{j} n_{j+1}\right\rangle$ in the CGGE, one-body NCGGE, trigonal NCGGE, two-body NCGGE, and long-time average with the initial state $\left|\psi_{\mathrm{ini}}^{\mathrm{A}}\right\rangle$ and $L=600, L_{\text {ini }}=360$, and $N=120$. There are no characteristic peaks which cannot be explained by the CGGE such as in the case of $\left|\psi_{\text {ini }}^{\mathrm{B}}\right\rangle$. (b) Error of GGEs $\left|\left\langle\hat{n}_{j} \hat{n}_{j+1}\right\rangle_{\mathrm{GGE}}-\left\langle\hat{n}_{j} \hat{n}_{j+1}\right\rangle_{\mathrm{LT}}\right|$ for the local density-density correlation between sites $j$ and $j+1$.

characteristic peak that survives in the thermodynamic limit, and the CGGE is thus accurate for density-density correlation with any pair of sites unlike the excited initial state $\left|\psi_{\text {ini }}^{\mathrm{B}}\right\rangle$. The difference between the two-body NCGGE and the long-time average is due to the accidental degeneracy.
[1] L. D'Alessio, Y. Kafri, A. Polkovnikov, and M. Rigol, Adv. Phys. 65, 239 (2016).

[2] J. Eisert, M. Friesdorf, and C. Gogolin, Nat. Phys. 11, 124 (2015).

[3] E. Ilievski, M. Medenjak, T. Prosen, and L. Zadnik, J. Stat. Mech. (2016) 064008.

[4] T. Mori, T. N. Ikeda, E. Kaminishi, and M. Ueda, J. Phys. B: At., Mol. Opt. Phys. 51, 112001 (2018).

[5] T. Kinoshita, T. Wenger, and D. S. Weiss, Nature (London) 440, 900 (2006).

[6] S. Trotzky, Y.-A. Chen, A. Flesch, I. P. McCulloch, U. Schollwöck, J. Eisert, and I. Bloch, Nat. Phys. 8, 325 (2011).
[7] T. Langen, R. Geiger, M. Kuhnert, B. Rauer, and J. Schmiedmayer, Nat. Phys. 9, 640 (2013).

[8] T. Langen, S. Erne, R. Geiger, B. Rauer, T. Schweigler, M. Kuhnert, W. Rohringer, I. E. Mazets, T. Gasenzer, and J. Schmiedmayer, Science 348, 207 (2015).

[9] M. Schreiber, S. S. Hodgman, P. Bordia, H. P. Lüschen, M. H. Fischer, R. Vosk, E. Altman, U. Schneider, and I. Bloch, Science 349, 842 (2015).

[10] A. M. Kaufman, M. E. Tai, A. Lukin, M. Rispoli, R. Schittko, P. M. Preiss, and M. Greiner, Science 353, 794 (2016).

[11] H. Tasaki, Phys. Rev. Lett. 80, 1373 (1998).

[12] P. Reimann, Phys. Rev. Lett. 101, 190403 (2008). 
[13] N. Linden, S. Popescu, A. J. Short, and A. Winter, Phys. Rev. E 79, 061103 (2009).

[14] J.M. Deutsch, Phys. Rev. A 43, 2046 (1991).

[15] M. Srednicki, Phys. Rev. E 50, 888 (1994).

[16] J. von Neumann, Z. Phys. 57, 30 (1929); [Eur. Phys. J. H 35, 201 (2010)].

[17] M. Rigol, V. Dunjko, and M. Olshanii, Nature (London) 452, 854 (2008).

[18] L. F. Santos and M. Rigol, Phys. Rev. E 81, 036206 (2010).

[19] H. Kim, T. N. Ikeda, and D. A. Huse, Phys. Rev. E 90, 052105 (2014).

[20] W. Beugeling, R. Moessner, and M. Haque, Phys. Rev. E 89, 042112 (2014).

[21] R. Steinigeweg, A. Khodja, H. Niemeyer, C. Gogolin, and J. Gemmer, Phys. Rev. Lett. 112, 130403 (2014).

[22] A. Khodja, R. Steinigeweg, and J. Gemmer, Phys. Rev. E 91, 012120 (2015).

[23] T. Yoshizawa, E. Iyoda, and T. Sagawa, Phys. Rev. Lett. 120, 200604 (2018).

[24] M. Rigol, V. Dunjko, V. Yurovsky, and M. Olshanii, Phys. Rev. Lett. 98, 050405 (2007).

[25] G. Biroli, C. Kollath, and A. M. Läuchli, Phys. Rev. Lett. 105, 250401 (2010).

[26] T. N. Ikeda, Y. Watanabe, and M. Ueda, Phys. Rev. E 87, 012125 (2013).

[27] V. Alba, Phys. Rev. B 91, 155123 (2015).

[28] A. Pal and D. A. Huse, Phys. Rev. B 82, 174411 (2010).

[29] S. Iyer, V. Oganesyan, G. Refael, and D. A. Huse, Phys. Rev. B 87, 134202 (2013).

[30] M. van den Worm, B. C. Sawyer, J. J. Bollinger, and M. Kastner, New J. Phys. 15, 083007 (2013).

[31] N. Shiraishi and T. Mori, Phys. Rev. Lett. 119, 030601 (2017).

[32] R. Mondaini, K. Mallayya, L. F. Santos, and M. Rigol, Phys. Rev. Lett. 121, 038901 (2018).

[33] E. T. Jaynes, Phys. Rev. 106, 620 (1957).

[34] L. Vidmar and M. Rigol, J. Stat. Mech. (2016) 064007.

[35] J.-S. Caux and R. M. Konik, Phys. Rev. Lett. 109, 175301 (2012).

[36] B. Pozsgay, J. Stat. Mech.: Theory Exp. (2013) P07003.
[37] M. Fagotti, M. Collura, F. H. L. Essler, and P. Calabrese, Phys. Rev. B 89, 125101 (2014).

[38] B. Wouters, J. De Nardis, M. Brockmann, D. Fioretto, M. Rigol, and J.-S. Caux, Phys. Rev. Lett. 113, 117202 (2014).

[39] B. Pozsgay, M. Mestyán, M. A. Werner, M. Kormos, G. Zaránd, and G. Takacs, Phys. Rev. Lett. 113, 117203 (2014).

[40] E. Ilievski, J. De Nardis, B. Wouters, J.-S. Caux, F. H. L. Essler, and T. Prosen, Phys. Rev. Lett. 115, 157201 (2015).

[41] R. Hamazaki, T. N. Ikeda, and M. Ueda, Phys. Rev. E 93, 032116 (2016).

[42] J. Cardy, J. Stat. Mech.: Theory Exp. (2016) 023103.

[43] M. Kollar and M. Eckstein, Phys. Rev. A 78, 013626 (2008).

[44] M. Kollar, F. A. Wolf, and M. Eckstein, Phys. Rev. B 84, 054304 (2011).

[45] T. Caneva, E. Canovi, D. Rossini, G. E. Santoro, and A. Silva, J. Stat. Mech. (2011) P07015.

[46] K. He, L. F. Santos, T. M. Wright, and M. Rigol, Phys. Rev. A 87, 063637 (2013).

[47] S. Ziraldo, A. Silva, and G. E. Santoro, Phys. Rev. Lett. 109, 247205 (2012).

[48] S. Ziraldo and G. E. Santoro, Phys. Rev. B 87, 064201 (2013).

[49] X. Wang and P. Tong, J. Stat. Mech. 2017, 113107 (2017).

[50] E. Kaminishi, T. Mori, T. N. Ikeda, and M. Ueda, Nat. Phys. 11, 1050 (2015).

[51] M. Fagotti, J. Stat. Mech. (2014) P03016.

[52] V. I. Yukalov, Phys. Lett. A 375, 2797 (2011).

[53] A. J. Short, New J. Phys. 13, 053009 (2011).

[54] P. Reimann and M. Kastner, New J. Phys. 14, 043020 (2012).

[55] A. C. Cassidy, C. W. Clark, and M. Rigol, Phys. Rev. Lett. 106, 140405 (2011).

[56] M. Mierzejewski and L. Vidmar, Phys. Rev. Lett. 124, 040603 (2020).

[57] N. Yunger Halpern, P. Faist, J. Oppenheim, and A. Winter, Nat. Commun. 7, 12051 (2016).

[58] N. Yunger Halpern, M. E. Beverland, and A. Kalev, Phys. Rev. E 101, 042117 (2020).

[59] S. Golden, Phys. Rev. 137, B1127 (1965).

[60] T. N. Ikeda and M. Ueda, Phys. Rev. E 92, 020102(R) (2015). 